\title{
Equivalence à la Mundici for commutative lattice-ordered monoids
}

\author{
Marco Abbadini
}

\begin{abstract}
We provide a generalization of Mundici's equivalence between unital Abelian lattice-ordered groups and MV-algebras: the category of unital commutative lattice-ordered monoids is equivalent to the category of $M V$-monoidal algebras. Roughly speaking, unital commutative latticeordered monoids are unital Abelian lattice-ordered groups without the unary operation $x \mapsto-x$. The primitive operations are $+, \vee, \wedge, 0,1,-1$. A prime example of these structures is $\mathbb{R}$, with the obvious interpretation of the operations. Analogously, MV-monoidal algebras are MV-algebras without the negation $x \mapsto \neg x$. The primitive operations are $\oplus, \odot, \vee, \wedge$, 0,1 . A motivating example of MV-monoidal algebra is the negation-free reduct of the standard MV-algebra $[0,1] \subseteq \mathbb{R}$. We obtain the original Mundici's equivalence as a corollary of our main result.
\end{abstract}

Mathematics Subject Classification. 06F05, 54F05, 03 C05.

Keywords. Lattice-ordered monoids, Lattice-ordered groups, Categorical equivalence, MV-algebras, Compact ordered spaces, Continuous orderpreserving functions.

\section{Introduction}

In [16], Mundici proved that the category of unital Abelian lattice-ordered groups (unital Abelian $\ell$-groups, for short) is equivalent to the category of MV-algebras. In Theorem 8.21, our main result, we establish the following generalization: The category of unital commutative lattice-ordered monoids is equivalent to the category of $M V$-monoidal algebras.

Roughly speaking, unital commutative lattice-ordered monoids (unital commutative $\ell$-monoids, for short) are unital Abelian $\ell$-groups without the unary operation $x \mapsto-x$, whereas MV-monoidal algebras are MV-algebras without the negation $x \mapsto \neg x$ (precise definitions will be given in Section 2).

Presented by P. Jipsen. 
The operations of unital commutative $\ell$-monoids are $+, \vee, \wedge, 0,1,-1$, whereas the operations of $\mathrm{MV}$-monoidal algebras are $\oplus, \odot, \vee, \wedge, 0,1$. A motivating example of unital commutative $\ell$-monoid is $\mathbb{R}$, with the obvious interpretation of the operations, whereas a motivating example of MV-monoidal algebra is the negation-free reduct of the standard MV-algebra [0,1]. Furthermore, for every topological space $X$ equipped with a preorder, the set of bounded continuous order-preserving functions from $X$ to $\mathbb{R}$ is an example of a unital commutative $\ell$-monoid, whereas the set of continuous order-preserving functions from $X$ to $[0,1]$ is an example of an MV-monoidal algebra. The author's interest for unital commutative $\ell$-monoids originated from these last examples, as we now illustrate in some detail.

Given a compact Hausdorff space $X$, the set $C(X, \mathbb{R})$ of continuous functions from $X$ to $\mathbb{R}$ is a divisible Archimedean unital Abelian $\ell$-group, complete in the uniform metric. In fact, we have a duality between the category CompHaus of compact Hausdorff spaces and continuous maps and the category $\bar{G}$ of divisible Archimedean metrically complete unital Abelian $\ell$-groups (see $[21,20])$. Similarly, we may consider on the set $C(X,[0,1])$ of continuous functions from $X$ to $[0,1]$ pointwise-defined operations inherited from $[0,1]$; for example, the operations of MV-algebras. Developing this idea, one can show that CompHaus is dually equivalent to a variety $\Delta$ of (infinitary) algebras (see $[9,11,14])$. These algebras can be thought of as MV-algebras with an additional operation of countably infinite arity satisfying some additional axioms. In fact, we have an equivalence between $\bar{G}$ and $\Delta$, which is essentially a restriction of the equivalence between unital Abelian $\ell$-groups and MV-algebras.

A compact ordered space is a compact space $X$ endowed with a partial order $\leqslant$ on $X$ so that the set $\{(x, y) \in X \times X \mid x \leqslant y\}$ is closed in $X \times X$ with respect to the product topology. This notion was introduced by Nachbin [17]. If we replace compact Hausdorff spaces by compact ordered spaces in the aforementioned discussion involving CompHaus, $\Delta$ and $\bar{G}$, then we may accordingly replace Mundici's equivalence with our Theorem 8.21. Given a compact ordered space $X$, let us consider the set $C_{\leqslant}(X,[0,1])$ of continuous order-preserving functions from $X$ to $[0,1]$ : we can endow $C_{\leqslant}(X,[0,1])$ with pointwise-defined operations $\oplus, \odot, \vee, \wedge, 0,1$ (which are the operations of MV-monoidal algebras). Pursuing a similar idea, in [10] it was proved that the category of compact ordered spaces and continuous order-preserving maps is dually equivalent to a quasi-variety of infinitary algebras $([1,3]$ show that this quasi-variety actually is a variety). However, the operations are somewhat unwieldy, and one might want to investigate the set $C_{\leqslant}(X, \mathbb{R})$ of continuous order-preserving real-valued functions, instead. In fact, $C_{\leqslant}(X, \mathbb{R})$ is a unital commutative $\ell$-monoid. The main motivation of this paper is to make the connection between $C_{\leqslant}(X, \mathbb{R})$ (unital commutative $\ell$-monoids) and $C_{\leqslant}(X,[0,1])$ (MV-monoidal algebras) explicit.

There are both pros and cons in working with unital commutative $\ell$ monoids or MV-monoidal algebras. On the one hand, it is easier to work with the axioms of unital commutative $\ell$-monoids rather than those of MV-monoidal algebras. On the other hand, the category of MV-monoidal algebras is a variety 
of finitary algebras axiomatized by a finite number of equations, so the tools of universal algebra apply. The equivalence established here allows transferring the pros of one category to the other one.

Our result specializes to Mundici's equivalence between unital Abelian $\ell$-groups and MV-algebras (Appendix A). We remark that, in contrast to the proof of Mundici's equivalence in [16], we do not use the axiom of choice to prove the equivalence between unital commutative $\ell$-monoids and MVmonoidal algebras.

We sketch the proof of our main result, Theorem 8.21. To obtain an equivalence

$$
\mathrm{u} \ell \mathrm{M} \underset{\tilde{\Xi}}{\stackrel{\tilde{\Gamma}}{\rightleftarrows}} \text { MVM }
$$

between the category $\mathrm{u} \ell \mathrm{M}$ of unital commutative $\ell$-monoids and the category MVM of MV-monoidal algebras, we show that we have two equivalences

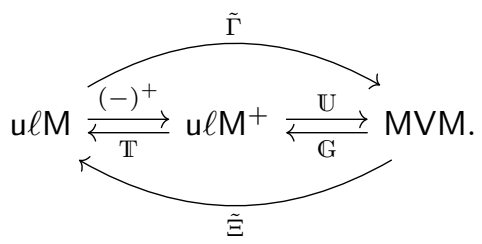

Here $\mathrm{u} \ell \mathrm{M}^{+}$is the category of 'positive-unital commutative $\ell$-monoids' (Definition 4.1), which are the positive cones of unital commutative $\ell$-monoids. The functor $\tilde{\Gamma}$ maps a unital commutative $\ell$-monoid $M$ to its 'unit interval' $\tilde{\Gamma}(M)$ (Section 3). We construct a quasi-inverse in two steps. As a first step, given an MV-monoidal algebra $A$, we define the set $\mathbb{G}(A)$ of ' $\operatorname{good}$ sequences in $A^{\prime}$ (Section 5), and we equip this set with the structure of a positive-unital commutative $\ell$-monoid (Section 7). As a second step, we consider translations of the elements of $\mathbb{G}(A)$ by negative integers; in this way we obtain a unital commutative $\ell$-monoid $\mathrm{TG}(A)$, where $\mathrm{T}: \mathrm{u} \ell \mathrm{M}^{+} \rightarrow \mathrm{u} \ell \mathrm{M}$ is a functor (Section 4). To show that the composition of these two steps provides a quasi-inverse of $\tilde{\Gamma}$, we write $\tilde{\Gamma}$ as the composite of two functors $(-)^{+}$and $\mathbb{U}$. The functor $(-)^{+}$associates to $M$ its 'positive cone' $M^{+}$; the functor $\mathbb{U}$ associates to $M^{+}$ its unit interval. We will show that $(-)^{+}$and $\mathrm{T}$ are quasi-inverses (Section 4 ), and that $\mathbb{U}$ and $\mathbb{G}$ are quasi-inverses (Section 8 ); from this, it follows that $\tilde{\Gamma}$ and $\tilde{\Xi}:=\mathbb{T} G$ are quasi-inverses, and hence the categories of unital commutative $\ell$-monoids and MV-monoidal algebras are equivalent (Theorem 8.21).

By the time of publication of the present paper, the main result (Theorem 8.21) has appeared also in the author's Ph.D. thesis [2, Chapter 4]. However, the proofs are different. In [2], the author uses Birkhoff's subdirect representation theorem, which simplifies the arguments but relies on the axiom of choice. Moreover, while in this paper we construct a quasi-inverse of $\tilde{\Gamma}$ as the composite of two functors, in [2] a one-step construction is adopted. 
In Appendix B we show that subdirectly irreducible MV-monoidal algebras are totally ordered, and in Appendix $\mathrm{C}$ we show that every good sequence in a subdirectly irreducible MV-monoidal algebra is of the form $(1, \ldots, 1, x, 0,0, \ldots)$. Even if we do not use these last two results, we have included them because they seem of interest.

\section{The algebras}

\subsection{Unital commutative lattice-ordered monoids}

The set $\mathbb{R}$, endowed with the binary operations + (addition), $\vee$ (maximum), $\wedge$ (minimum), and the constants 0,1 and -1 is a prototypical example of $a$ unital commutative lattice-ordered monoid.

Definition 2.1. A commutative lattice-ordered monoid (shortened as commutative $\ell$-monoid) is an algebra $\langle M ;+, \vee, \wedge, 0\rangle$ (arities $2,2,2,0$ ) with the following properties.

(M1) $\langle M ; \vee, \wedge\rangle$ is a distributive lattice.

(M2) $\langle M ;+, 0\rangle$ is a commutative monoid.

(M3) + distributes over $\vee$ and $\wedge$.

Definition 2.2. A unital commutative lattice-ordered monoid (unital commutative $\ell$-monoid, for short) is an algebra $\langle M ;+, \vee, \wedge, 0,1,-1\rangle$ (arities $2,2,2$, $0,0,0)$ with the following properties.

(U0) $\langle M ;+, \vee, \wedge, 0\rangle$ is a commutative $\ell$-monoid.

(U1) $-1+1=0$.

(U2) $0 \leqslant 1$.

(U3) For every $x \in M$ there exists $n \in \mathbb{N}$ such that

$$
\underbrace{(-1)+\cdots+(-1)}_{n \text { times }} \leqslant x \leqslant \underbrace{1+\cdots+1}_{n \text { times }} .
$$

The element 1 is called the positive unit, and the element -1 is called the negative unit.

We warn the reader that some authors do not assume the lattice to be distributive, nor that + distributes over both $\wedge$ and $\vee$.

We denote with $\mathrm{u} \ell \mathrm{M}$ the category of unital commutative $\ell$-monoids with homomorphisms. We write $z-1$ for $z+(-1)$. Given $n \in \mathbb{N}$, we write $n$ for $\underbrace{1+\cdots+1}_{n \text { times }}$ and $-n$ for $\underbrace{(-1)+\cdots+(-1)}_{n \text { times }}$.

Remark 2.3. Given a unital commutative $\ell$-monoid $\langle M ;+, \vee, \wedge, 0,1,-1\rangle$, we define the operation $x \cdot y:=x-1+y$. This operation does not coincide on $\mathbb{R}$ with the usual multiplication. However, we still use this notation because the equations $x \cdot 1=x=1 \cdot x$ hold. In fact, unital commutative $\ell$-monoids admit a term-equivalent description in the signature $\{+, \cdot, \vee, \wedge, 0,1\}$, from which the constant -1 can be recast as $0 \cdot 0$. In this signature, Axioms U0 and U1 are equivalent to: 
(E1) $\langle M ; \vee, \wedge\rangle$ is a distributive lattice.

(E2) $\langle M ;+, 0\rangle$ and $\langle M ; \cdot, 1\rangle$ are commutative monoids.

(E3) Both the operations + and $\cdot$ distribute over both $\vee$ and $\wedge$.

(E4) $(x \cdot y)+z=x \cdot(y+z)$.

(E5) $(x+y) \cdot z=x+(y \cdot z)$.

(Note that Axioms E4 and E5 are equivalent, given the commutativity of + and $\cdot$.) The addition of Axiom U2 equals the addition of the following axiom.

(E5) $0 \leqslant 1$.

The addition of Axiom U3 equals the addition of the following axiom.

(E6) For every $x \in M$ there exists $n \in \mathbb{N}$ such that

$$
\underbrace{0 \cdots \cdots 0}_{n \text { times }} \leqslant x \leqslant \underbrace{1+\cdots+1}_{n \text { times }} .
$$

The class of algebras satisfying Axioms E1-E6 is term-equivalent to the class of unital commutative $\ell$-monoids. One interesting thing about Axioms E1-E6 is that their symmetries resemble the ones in the definition of MVmonoidal algebras below. We will use Axioms E1-E6 to explain the axioms of MV-monoidal algebras below; besides this usage, we will stick to Axioms U0-U3 throughout the paper.

Example 2.4. For every topological space $X$ equipped with a preorder, the set of bounded continuous order-preserving functions from $X$ to $\mathbb{R}$ is a unital commutative $\ell$-monoid.

\subsection{MV-monoidal algebras}

In the following, we define the variety MVM of MV-monoidal algebras, which are finitary algebras axiomatised by a finite number of equations. Our main result is that the categories $\mathrm{u} \ell \mathrm{M}$ and MVM are equivalent. Without giving the details now, we anticipate the fact that the equivalence is given by the functor $\tilde{\Gamma}: \mathrm{u} \ell \mathrm{M} \rightarrow \mathrm{MVM}$ that maps a unital commutative $\ell$-monoid $M$ to the set $\{x \in M \mid 0 \leqslant x \leqslant 1\}$, endowed with the operations $\oplus, \odot, \wedge, \vee, 0$ and 1 , where $\wedge, \vee, 0$ and 1 are defined by restriction, and $\oplus$ and $\odot$ are defined by $x \oplus y:=(x+y) \wedge 1$ and $x \odot y:=(x+y-1) \vee 0$.

On $[0,1]$, consider the elements 0 and 1 and the operations $x \vee y:=\max \{x, y\}$, $x \wedge y:=\min \{x, y\}, x \oplus y:=\min \{x+y, 1\}$, and $x \odot y:=\max \{x+y-1,0\}$. This gives a prime example of what we call an MV-monoidal algebra.

Definition 2.5. An $M V$-monoidal algebra is an algebra $\langle A ; \oplus, \odot, \vee, \wedge, 0,1\rangle$ (arities $2,2,2,2,0,0)$ satisfying the following equational axioms.

(A1) $\langle A ; \vee, \wedge\rangle$ is a distributive lattice.

(A2) $\langle A ; \oplus, 0\rangle$ and $\langle A ; \odot, 1\rangle$ are commutative monoids.

(A3) Both the operations $\oplus$ and $\odot$ distribute over both $\vee$ and $\wedge$.

(A4) $(x \oplus y) \odot((x \odot y) \oplus z)=(x \odot(y \oplus z)) \oplus(y \odot z)$.

(A5) $(x \odot y) \oplus((x \oplus y) \odot z)=(x \oplus(y \odot z)) \odot(y \oplus z)$.

(A6) $(x \odot y) \oplus z=((x \oplus y) \odot((x \odot y) \oplus z)) \vee z$.

(A7) $(x \oplus y) \odot z=((x \odot y) \oplus((x \oplus y) \odot z)) \wedge z$. 
Before commenting on the axioms, we remark that Axioms A4 and A5 are equivalent, given the commutativity of $\oplus$ and $\odot$. We have included both so to make it clear that, if $\langle A ; \oplus, \odot, \vee, \wedge, 0,1\rangle$ is an MV-monoidal algebra, then also the 'dual' algebra $\langle A ; \odot, \oplus, \wedge, \vee, 1,0\rangle$ is an MV-monoidal algebra.

Axioms A1-A3 coincide with Axioms E1-E3 in Remark 2.3. So, in a sense, the difference between MV-monoidal algebras and unital commutative $\ell$-monoids lies in the difference bewteen the conjunction of Axioms A4-A7 and the conjunction of Axioms E4-E6. We mention here that 0 and 1 are bounds of the underlying lattice of an MV-monoidal algebra (Lemma 6.5); this fact is not completely obvious, given that the proof makes use of almost all the axioms of MV-monoidal algebras.

Axiom A4 is a sort of associativity, which resembles Axiom E4, i.e. $(x$. $y)+z=x \cdot(y+z)$. In particular, one verifies that the interpretation on $[0,1]$ of both the left-hand and right-hand side of Axiom A4 equals

$$
((x+y+z-1) \vee 0) \wedge 1 .
$$

Notice that the element $x+y+z-1$ appearing in (2.1) coincides, using the definition of $\cdot$ from Remark 2.3, with the interpretation on $\mathbb{R}$ of $(x \cdot y)+z$ and $x \cdot(y+z)$. In fact, Axiom A4 is essentially the condition $(x \cdot y)+z=x \cdot(y+z)$ expressed at the unital level, i.e.:

$$
(((x \cdot y)+z) \vee 0) \wedge 1=((x \cdot(y+z)) \vee 0) \wedge 1
$$

Indeed, the presence of the term $x \cdot y$ in the left-hand side of (2.2) corresponds to the presence of the terms $x \oplus y$ and $x \odot y$ in the left-hand side of Axiom A4, and the presence of the term $y+z$ in the right-hand side of (2.2) corresponds to the presence of the terms $y \oplus z$ and $y \odot z$ in the right-hand side of Axiom A4.

Analogously, Axiom A5 corresponds to Axiom E5, i.e. $(x+y) \cdot z=x+$ $(y \cdot z)$.

Axiom A6 expresses how the term $(x \odot y) \oplus z$ differs from its non-truncated version $(x \cdot y)+z$ : essentially, the axiom can be read as

$$
(x \odot y) \oplus z=((x \cdot y)+z) \vee z .
$$

Analogously, Axiom A7 can be read as

$$
(x \oplus y) \odot z=((x+y) \cdot z) \wedge z .
$$

We remark that MV-monoidal algebras form a variety of algebras whose primitive operations are finitely many and of finite arity, and which is axiomatised by a finite number of equations. We let MVM denote the category of MV-monoidal algebras with homomorphisms.

Remark 2.6. Bounded distributive lattices form a subvariety of the variety of MV-monoidal algebras, obtained by adding the axioms $x \oplus y=x \vee y$ and $x \odot y=x \wedge y$. 


\section{The unit interval functor}

In this section we define a functor $\tilde{\Gamma}$ from the category of unital commutative $\ell$-monoids to the category of MV-monoidal algebras; the main goal of the paper is to show that $\tilde{\Gamma}$ is an equivalence. For a unital commutative $\ell$-monoid $M$, we set $\tilde{\Gamma}(M):=\{x \in M \mid 0 \leqslant x \leqslant 1\}$. We will endow $\tilde{\Gamma}(M)$ with a structure of an MV-monoidal algebra. Clearly, $0,1 \in \tilde{\Gamma}(M)$. Moreover, we define $\vee$ and $\wedge$ on $\tilde{\Gamma}(M)$ by restriction. Finally, for $x, y \in \tilde{\Gamma}(M)$, we set

$$
x \oplus y:=(x+y) \wedge 1
$$

and

$$
x \odot y:=(x+y-1) \vee 0,
$$

To see that $\oplus$ and $\odot$ are internal operations on $\tilde{\Gamma}(M)$, we make use of the following.

Lemma 3.1. Let $M$ be a commutative $\ell$-monoid. For all $x, y, x^{\prime}, y^{\prime} \in M$ such that $x \leqslant x^{\prime}$ and $y \leqslant y^{\prime}$, we have $x+y \leqslant x^{\prime}+y^{\prime}$.

Proof. Since + distributes over $\vee$, we have $(x+y) \vee\left(x+y^{\prime}\right)=x+\left(y \vee y^{\prime}\right)=x+y^{\prime}$. Therefore, $x+y \leqslant x+y^{\prime}$; analogously, $x+y^{\prime} \leqslant x^{\prime}+y^{\prime}$. Hence, $x+y \leqslant x+y^{\prime} \leqslant$ $x^{\prime}+y^{\prime}$.

By Lemma 3.1, $\oplus$ and $\odot$ are internal operations on $\tilde{\Gamma}(M)$ : indeed, the condition $x \oplus y \in \tilde{\Gamma}(M)$ holds because $x+y \geqslant 0+0=0$, and the condition $x \odot y \in \tilde{\Gamma}(M)$ holds because $x+y-1 \leqslant 1+1-1=1$.

Our next goal-met in Proposition 3.6 below - is to show that $\tilde{\Gamma}(M)$ is an MV-monoidal algebra. We need some lemmas.

Lemma 3.2. Let $M$ be a unital commutative $\ell$-monoid, and let $x, y, z \in \tilde{\Gamma}(M)$. Then

$$
(x \odot y) \oplus z=((x+y+z-1) \vee z) \wedge 1,
$$

and

$$
(x \oplus y) \odot z=((x+y+z-1) \wedge z) \vee 0 .
$$

Proof. We have

$$
\begin{aligned}
(x \odot y) \oplus z & =((x \odot y)+z) \wedge 1 & & (\text { def. of } \oplus) \\
& =(((x+y-1) \vee 0)+z) \wedge 1 & & (\text { def. of } \odot) \\
& =((x+y+z-1) \vee z) \wedge 1 & & (+ \text { distr. over } \wedge)
\end{aligned}
$$

and

$$
\begin{aligned}
(x \oplus y) \odot z & =((x \oplus y)+z-1) \vee 0 & & (\text { def. of } \odot) \\
& =(((x+y) \wedge 1)+z-1) \vee 0 & & (\text { def. of } \oplus) \\
& =((x+y+z-1) \wedge z) \vee 0 . & & (+ \text { distr. over } \wedge)
\end{aligned}
$$


Lemma 3.3. For all $x$ and $y$ in a commutative $\ell$-monoid we have

$$
(x \wedge y)+(x \vee y)=x+y \text {. }
$$

Proof. We recall the proof, available in [6], of the two inequalities:

$$
\begin{aligned}
(x \wedge y)+(x \vee y) & =((x \wedge y)+x) \vee((x \wedge y)+y) \\
& \leqslant(y+x) \vee(x+y)=x+y ; \\
(x \wedge y)+(x \vee y) & =(x+(x \vee y)) \wedge(y+(x \vee y)) \\
& \geqslant(x+y) \wedge(y+x)=x+y .
\end{aligned}
$$

Lemma 3.4. Let $M$ be a unital commutative $\ell$-monoid, and let $x, y \in \tilde{\Gamma}(M)$. Then

$$
(x \oplus y)+(x \odot y)=x+y
$$

Proof. We have

$$
\begin{aligned}
(x \oplus y)+(x \odot y) & =((x+y) \wedge 1)+((x+y-1) \vee 0) & & (\text { def. of } \oplus \text { and } \odot) \\
& =((x+y) \wedge 1)+((x+y) \vee 1)-1 & & (+ \text { distr. over } \vee) \\
& =x+y+1-1 & & \text { (Lemma 3.3) } \\
& =x+y . & &
\end{aligned}
$$

Lemma 3.5. Let $M$ be a unital commutative $\ell$-monoid. For all $x, y, z \in \tilde{\Gamma}(M)$, the elements $(x \oplus y) \odot((x \odot y) \oplus z),(x \odot y) \oplus((x \oplus y) \odot z),(x \odot(y \oplus z)) \oplus(y \odot z)$, and $(x \oplus(y \odot z)) \odot(y \oplus z)$ coincide with

$$
(x+y+z-1) \vee 0) \wedge 1 .
$$

Proof. We have

$$
\begin{aligned}
(x & \oplus y) \odot((x \odot y) \oplus z) & & \\
& =(((x \oplus y)+(x \odot y)+z) \wedge(x \oplus y)) \vee 0 & & (\text { Lemma 3.2) } \\
& =((x+y+z-1) \wedge(x \oplus y)) \vee 0 & & (\text { Lemma 3.4) } \\
& =((x+y+z-1) \wedge(x+y) \wedge 1) \vee 0 & & (\text { def. of } \oplus) \\
& =((x+y+z-1) \wedge 1) \vee 0 & & (x+y+z-1 \leqslant x+y) \\
& =((x+y+z-1) \vee 0) \wedge 1 . & &
\end{aligned}
$$

and

$$
\begin{aligned}
(x & \odot(y \oplus z)) \oplus(y \odot z) & & \\
& =((x+(y \oplus z)+(y \odot z)) \vee(y \odot z)) \wedge 1 & & (\text { Lemma 3.2 }) \\
& =((x+y+z-1) \vee(y \odot z)) \wedge 1 & & (\text { Lemma 3.4) } \\
& =((x+y+z-1) \vee(y+z-1) \vee 0) \wedge 1 & & (\text { def. of } \odot) \\
& =((x+y+z-1) \vee 0) \wedge 1 . & & (x+y+z-1 \geqslant y+z-1)
\end{aligned}
$$

The fact that also $(x \odot y) \oplus((x \oplus y) \odot z)$ and $(x \oplus(y \odot z)) \odot(y \oplus z)$ coincide with $(x+y+z-1) \vee 0) \wedge 1$ follows from the commutativity of $\oplus$ and $\odot$ (which is easily seen to hold) and the commutativity of + . 
Proposition 3.6. Let $M$ be a unital commutative $\ell$-monoid. Then $\tilde{\Gamma}(M)$ is an MV-monoidal algebra.

Proof. Axioms A1-A3 are obtained by straightforward computations. Axioms A4 and A5 hold by Lemma 3.5. Axioms A6 and A7 hold by Lemmas 3.2 and 3.5 .

Given a morphism of unital commutative $\ell$-monoids $f: M \rightarrow N$, we denote with $\tilde{\Gamma}(f)$ its restriction $\tilde{\Gamma}(f): \tilde{\Gamma}(M) \rightarrow \tilde{\Gamma}(N)$. This establishes a functor

$$
\tilde{\Gamma}: \mathrm{u} \ell \mathrm{M} \rightarrow \mathrm{MVM} \text {. }
$$

Our main goal is to show that $\tilde{\Gamma}$ is an equivalence of categories.

\section{Positive cones}

In [7, Chapter 2], the authors proceed in two steps to prove that, for an MValgebra $A$, there exists a unital Abelian $\ell$-group that envelops $A$. First, a partially ordered monoid $M_{A}$ is constructed from $A$. Then a unital Abelian $\ell$-group $G_{A}$ is defined (in a way that is analogous to the definition of $\mathbb{Z}$ from $\mathbb{N}$ ). In this paper, we proceed analogously: the role of $A$ is played by MVmonoidal algebras, the role of $G_{A}$ is played by unital commutative $\ell$-monoids, and the role of $M_{A}$ is played by what we call positive-unital commutative $\ell$ monoids. Roughly speaking, if we think of a unital commutative $\ell$-monoid as the interval $(-\infty, \infty)$, then an MV-monoidal algebra is the interval $[0,1]$, whereas a positive-unital commutative $\ell$-monoid is the interval $[0, \infty)$.

To prove that $\tilde{\Gamma}$ is an equivalence, we show that $\tilde{\Gamma}$ is the composite of two equivalences

$$
\mathrm{u} \ell \mathrm{M} \stackrel{(-)^{+}}{\longrightarrow} \mathrm{u} \ell \mathrm{M}^{+} \stackrel{\mathrm{U}}{\longrightarrow} \mathrm{MVM}
$$

where $\mathrm{u} \ell \mathrm{M}^{+}$is the category - yet to be defined - of positive-unital commutative $\ell$-monoids. The idea is that, for $M \in \mathrm{u} \ell \mathrm{M}$, we have $M^{+}:=\{x \in M \mid x \geqslant 0\}$, and for $N \in \mathrm{u} \ell \mathrm{M}^{+}$, we have $\mathbb{U}(N):=\{x \in N \mid x \leqslant 1\}$, so that $\mathbb{U}\left(M^{+}\right)=\{x \in$ $M \mid 0 \leqslant x \leqslant 1\}=\tilde{\Gamma}(M)$. In this section, we define the functor $(-)^{+}$, and we exhibit a quasi-inverse $\mathbb{T}$. We remark that one could construct a quasi-inverse functor for $\tilde{\Gamma}$ just in one step: see the author's Ph.D. thesis [2, Chapter 4] for the employment of this approach.

Given a unital commutative $\ell$-monoid $M$, we set $M^{+}:=\{x \in M \mid x \geqslant 0\}$. With the following definition, we aim to capture the structure of $M^{+}$for $M$ a unital commutative $\ell$-monoid.

Definition 4.1. By a positive-unital commutative $\ell$-monoid we mean an algebra $\langle M ;+, \vee, \wedge, 0,1,-\ominus 1\rangle$ (arities $2,2,2,0,0,1)$ such that, for every $x \in M$, the following properties hold.

(P0) $\langle M ;+, \vee, \wedge, 0\rangle$ is a commutative $\ell$-monoid.

(P1) $x \geqslant 0$.

(P2) $(x+1) \ominus 1=x$. 
(P3) $(x \ominus 1)+1=x \vee 1$.

(P4) There exists $n \in \mathbb{N}$ such that $x \leqslant \underbrace{1+\cdots+1}_{n \text { times }}$.

We denote with $\mathrm{u} \ell \mathrm{M}^{+}$the category of positive-unital commutative $\ell$ monoids with homomorphisms. Given $n \in \mathbb{N}$, we write $n$ for $\underbrace{1+\cdots+1}_{n \text { times }}$.

In this section, we show that $\mathrm{u} \ell \mathrm{M}$ and $\mathrm{u} \ell \mathrm{M}^{+}$are equivalent.

Lemma 4.2. Let $M$ be a positive-unital commutative $\ell$-monoid. For all $x, y \in$ $M$ and every $n \in \mathbb{N}$, if $x+n=y+n$ then $x=y$.

Proof. The proof proceeds by induction on $n \in \mathbb{N}$. The case $n=0$ is trivial. Suppose the statement holds for $n \in \mathbb{N}$. If $x+(n+1)=y+(n+1)$, then

$$
x+n \stackrel{\text { Axiom }}{=} \mathrm{P} 2(x+n+1) \ominus 1=(y+n+1) \ominus 1 \stackrel{\text { Axiom } \mathrm{P} 2}{=} y+n,
$$

and then, by the inductive hypothesis, $x=y$.

Remark 4.3. Let $x$ and $y$ be elements of a positive-unital commutative $\ell$ monoid. Then

$$
y=x \ominus 1 \stackrel{\text { Lemma }^{4.2}}{\Longleftrightarrow} y+1=(x \ominus 1)+1 \stackrel{\text { Axiom }}{\Longleftrightarrow} \text { P3 } y+1=x \vee 1 .
$$

Moreover,

$$
y=0 \stackrel{\text { Lemma }}{\Longleftrightarrow}{ }^{4.2} y+1=1 .
$$

This shows that the unary operation $-\ominus 1$ and the constant 0 can be explicitly defined from $\{\vee, \wedge, 1,+\}$. Therefore, every function $f: M \rightarrow N$ between positive-unital commutative $\ell$-monoids that preserves $+, \vee, \wedge$ and 1 preserves also $-\ominus 1$ and 0 , and hence it is a homomorphism.

Given a unital commutative $\ell$-monoid $M$, we endow $M^{+}$with the operations $+, \vee, \wedge, 0,1$ defined by restriction and with $-\ominus 1$ defined by $x \ominus 1:=(x-1) \vee 0$. The restriction of + on $M^{+}$is well-defined by Lemma 3.1 ; it is immediate that $\vee, \wedge$ and $-\ominus 1$ are well-defined, and that $0,1 \in M^{+}$.

Proposition 4.4. For every unital commutative $\ell$-monoid $M$, the algebra $M^{+}$ is a positive-unital commutative $\ell$-monoid.

Proof. The algebra $\left\langle M^{+} ;+, \vee, \wedge, 0\right\rangle$ is a commutative $\ell$-monoid because it is a subalgebra of the commutative $\ell$-monoid $\langle M ;+, \vee, \wedge, 0\rangle$; so, Axiom P0 holds. Axiom P1 holds by definition of $M^{+}$. Axiom P2 holds because, for every $x \in$ $M^{+}$, we have

$$
(x+1) \ominus 1=(x+1-1) \vee 0=x \vee 0=x .
$$

Axiom P3 holds because, for every $x \in M^{+}$, we have

$$
(x \ominus 1)+1=((x-1) \vee 0)+1=x \vee 1 .
$$

Axiom P4 holds because, by Axiom U3, for every $x \in M^{+}$there exists $n \in \mathbb{N}$ such that $x \leqslant n$. 
Given a morphism $f: M \rightarrow N$ of unital commutative $\ell$-monoids, $f$ restricts to a function $f^{+}$from $M^{+}$to $N^{+}$. Moreover, $f$ preserves $+, \vee, \wedge$ and 1 and so, by Remark 4.3, $f^{+}$is a morphism of positive-unital commutative $\ell$-monoids. This establishes a functor $(-)^{+}: \mathrm{u} \ell \mathrm{M} \rightarrow \mathrm{u} \ell \mathrm{M}^{+}$that maps $M$ to $M^{+}$, and maps a morphism $f: M \rightarrow N$ to its restriction $f^{+}: M^{+} \rightarrow N^{+}$. We will prove that $(-)^{+}$is an equivalence of categories (Theorem 4.16 below). To do so, we exhibit a quasi-inverse $\mathrm{T}: \mathrm{u} \ell \mathrm{M}^{+} \rightarrow \mathrm{u} \ell \mathrm{M}$.

Let $M$ be a positive-unital commutative $\ell$-monoid. We want to construct a unital commutative $\ell$-monoid $\mathrm{T}(M)$ such that, if $N$ is a unital commutative $\ell$ monoid and $N^{+} \cong M$, then $\mathrm{T}(M) \cong N$. Every element of a unital commutative $\ell$-monoid $N$ can be expressed as $x-n$ for some $x \in N^{+}$and $n \in \mathbb{N}$. Roughly speaking, we will obtain $\mathrm{T}\left(N^{+}\right) \cong N$ by translating the elements of $N^{+}$by negative integers. (In fact, $\mathrm{T}$ stands for 'translations'.)

Therefore, given a unital commutative $\ell$-monoid $M$, we consider the relation $\sim$ defined on $M \times \mathbb{N}$ as follows: $(x, n) \sim(y, m)$ if, and only if, $x+m=y+n$. Using Lemma 4.2 , it is not difficult to show that $\sim$ is an equivalence relation. The equivalence class of an element $(x, n)$ of $M \times \mathbb{N}$ is denoted by $[(x, n)]$, or simply $[x, n]$. We set $\mathbb{T}(M):=\frac{M \times \mathbb{N}}{\sim}$, and we endow $\mathbb{T}(M)$ with the operations of a unital commutative $\ell$-monoid:

$$
\begin{aligned}
0 & :=[0,0] ; \\
1 & :=[1,0] ; \\
-1 & :=[0,1] ; \\
{[x, n]+[y, m] } & :=[x+y, n+m] ; \\
{[x, n] \vee[y, m] } & :=[(x+m) \vee(y+n), n+m] ; \\
{[x, n] \wedge[y, m] } & :=[(x+m) \wedge(y+n), n+m] .
\end{aligned}
$$

Straightforward computations show that these operations are well-defined.

Remark 4.5. For every element $x$ of a positive-unital commutative $\ell$-monoid and all $n, m \in \mathbb{N}$ we have $(x, n) \sim(x+m, n+m)$.

Lemma 4.6. Let $M$ be a positive-unital commutative $\ell$-monoid. For all $x, y \in$ $M$ and every $n \in \mathbb{N}$ we have

$$
[x, n] \vee[y, n]=[x \vee y, n]
$$

and

$$
[x, n] \wedge[y, n]=[x \wedge y, n]
$$

Proof. We have

$$
\begin{aligned}
{[x, n] \vee[y, n] } & =[(x+n) \vee(y+n), n+n] \\
& =[(x \vee y)+n, n+n] \\
& =[x \vee y, n],
\end{aligned}
$$

and analogously for $\wedge$.

Proposition 4.7. For every positive-unital commutative $\ell$-monoid $M, \mathrm{~T}(M)$ is a unital commutative $\ell$-monoid. 
Proof. The fact that $\mathrm{T}(M)$ is a commutative monoid follows from the fact that $M$ and $\mathbb{N}$ are commutative monoids. Checking that $\mathbb{T}(M)$ is a distributive lattice is facilitated by Remark 4.5 and Lemma 4.6. Let us prove that + distributes over $\vee$ :

$$
\begin{aligned}
{[x, n]+([y, m] \vee[z, m]) } & =[x, n]+[y \vee z, m] \\
& =[x+(y \vee z), n+m] \\
& =[(x+y) \vee(x+z), n+m] \\
& =[x+y, n+m] \vee[x+z, n+m] \\
& =([x, n]+[y, m]) \vee([x, n]+[z, m]) .
\end{aligned}
$$

Analogously for $\wedge$. The axioms for 1 and -1 are easily seen to hold.

For a morphism of positive-unital commutative $\ell$-monoids $f: M \rightarrow N$, we set

$$
\begin{aligned}
\mathbb{T}(f): \mathbb{T}(M) & \longrightarrow \mathbb{T}(N) \\
{[x, n] } & \longmapsto[f(x), n] .
\end{aligned}
$$

The function $\mathrm{T}(f)$ is well-defined: indeed, if $(x, n) \sim(y, m)$, then $x+m=$ $y+n$, and then $f(x)+m=f(x+m)=f(y+n)=f(y)+n$, and therefore $(f(x), n) \sim(f(y), m)$. Moreover, $\mathbb{T}(f)$ is a morphism of unital commutative $\ell$-monoids. We show only that + is preserved:

$$
\begin{aligned}
\mathbb{T}(f)([x, n]+[y, m]) & =\mathbb{T}(f)([x+y, n+m]) \\
& =[f(x+y), n+m] \\
& =[f(x)+f(y), n+m] \\
& =[f(x), n]+[f(y), m] .
\end{aligned}
$$

One easily verifies that $\mathrm{T}: \mathrm{u} \ell \mathrm{M}^{+} \rightarrow \mathrm{u} \ell \mathrm{M}$ is a functor.

For each unital commutative $\ell$-monoid, we consider the function

$$
\begin{aligned}
\varepsilon_{M}^{0}: \mathbb{T}\left(M^{+}\right) & \longrightarrow M \\
{[x, n] } & \longmapsto x-n .
\end{aligned}
$$

The function $\varepsilon_{M}^{0}$ is well-defined: indeed, if $[x, n]=[y, m]$, then $x+m=y+n$ and therefore $x-n=y-m$.

Proposition 4.8. For every unital commutative $\ell$-monoid $M$, the function $\varepsilon_{M}^{0}$ : $\mathrm{T}\left(M^{+}\right) \rightarrow M$ is a morphism of unital commutative $\ell$-monoids.

Proof. The function $\varepsilon_{M}^{0}$ preserves 0,1 , and -1 because $\varepsilon_{M}^{0}([0,0])=0-0=0$, $\varepsilon_{M}^{0}([1,0])=1-0=1$ and $\varepsilon_{M}^{0}([0,1])=0-1=-1$. For all $x, y \in M^{+}$and $n \in \mathbb{N}$ we have

$$
\begin{aligned}
\varepsilon_{M}^{0}([x, n]+[y, m]) & =\varepsilon_{M}^{0}([x+y, n+m]) \\
& =(x+y)-(n+m) \\
& =(x-n)+(y-m) \\
& =\varepsilon_{M}^{0}([x, n])+\varepsilon_{M}^{0}([y, m]) .
\end{aligned}
$$



have

Hence, $\varepsilon_{M}^{0}$ preserves + . Moreover, for all $x, y \in M^{+}$and $n, m \in \mathbb{N}$, we

$$
\begin{array}{rlr}
\varepsilon_{M}^{0}([x, n] \vee[y, m]) & =\varepsilon_{M}^{0}([(x+m) \vee(y+n), n+m]) \\
& =((x+m) \vee(y+n))-(n+m) \\
& =(x+n) \vee(y+m) . \quad(+ \text { distr. over } \vee)
\end{array}
$$

Hence, $\varepsilon_{M}^{0}$ preserves $\vee$. Analogously, $\varepsilon_{M}^{0}$ preserves $\wedge$.

Proposition 4.9. $\varepsilon^{0}: \mathrm{T}(-)^{+} \dot{\rightarrow} 1_{\mathrm{u} \ell \mathrm{M}}$ is a natural transformation, i.e., for every morphism of unital commutative $\ell$-monoids $f: M \rightarrow N$, the following diagram commutes.

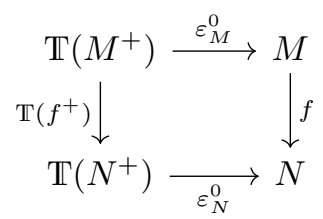

Proof. For every $x \in M^{+}$and every $n \in \mathbb{N}$ we have

$$
\begin{aligned}
\varepsilon_{N}^{0}\left(\mathbb{T}\left(f^{+}\right)([x, n])\right) & =\varepsilon_{N}^{0}\left(\left[f^{+}(x), n\right]\right) \\
& =\varepsilon_{N}^{0}([f(x), n]) \\
& =f(x)-n \\
& =f(x-n) \\
& =f\left(\varepsilon_{M}^{0}([x, n])\right) .
\end{aligned}
$$

Proposition 4.10. For every unital commutative $\ell$-monoid $M$, the function $\varepsilon_{M}^{0}: \mathbb{T}\left(M^{+}\right) \rightarrow M$ is bijective.

Proof. To prove injectivity, let $x, y \in M^{+}$, let $n, m \in \mathbb{N}$, and suppose we have $\varepsilon_{M}^{0}([x, n])=\varepsilon_{M}^{0}([y, m])$. Then,

$$
x-n=\varepsilon_{M}^{0}([x, n])=\varepsilon_{M}^{0}([y, m])=y-m ;
$$

hence $x+m=y+n$, and thus $[x, n]=[y, m]$; this proves injectivity. To prove surjectivity, for $x \in M$, choose $n \in \mathbb{N}$ such that $-n \leqslant x$; then $\varepsilon_{M}^{0}([x+n, n])=$ $x+n-n=x$.

For each positive-unital commutative $\ell$-monoid $M$, we consider the function

$$
\begin{aligned}
\eta_{M}^{0}: M & \longrightarrow(\mathrm{T}(M))^{+} \\
x & \longmapsto[x, 0] .
\end{aligned}
$$

Proposition 4.11. For every positive-unital commutative $\ell$-monoid $M$, the function $\eta_{M}^{0}: M \rightarrow(\mathbb{T}(M))^{+}$is a morphism of positive-unital commutative $\ell$ monoids.

Proof. It is easy to see that $\eta_{M}^{0}$ preserves $1,+, \vee$ and $\wedge$. Then, by Remark 4.3, the function $\eta_{M}^{0}$ is a morphism of positive-unital commutative $\ell$-monoids. 
Proposition 4.12. $\eta^{0}: 1_{\mathrm{u} \ell \mathrm{M}^{+}} \dot{\rightarrow}(-)^{+} \mathrm{T}$ is a natural transformation, i.e., for every morphism of positive-unital commutative $\ell$-monoids $f: M \rightarrow N$, the following diagram commutes.

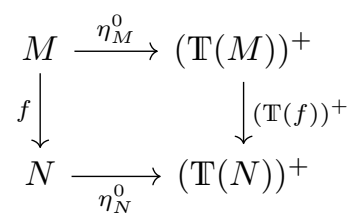

Proof. For every $x \in M$, we have

$$
\begin{aligned}
(\mathrm{T}(f))^{+}\left(\eta_{M}^{0}(m)\right) & =(\mathrm{T}(f))^{+}([x, 0])=\mathbb{T}(f)([x, 0]) \\
& =[f(x), 0]=\eta_{N}^{0}(f(x)) .
\end{aligned}
$$

Notation 4.13. Let $M$ be a positive-unital commutative $\ell$-monoid. We define, inductively on $n \in \mathbb{N}$, a function $(\cdot) \ominus n: M \rightarrow M$.

$$
\begin{aligned}
x \ominus 0:=x ; \\
(\text { for } n \geqslant 1) \quad x \ominus n=(x \ominus(n-1)) \ominus 1 .
\end{aligned}
$$

Lemma 4.14. Let $M$ be a positive-unital commutative $\ell$-monoid. For every $x \in$ $M$ and every $n \in \mathbb{N}$, we have

$$
(x \ominus n)+n=x \vee n .
$$

Proof. We prove the statement by induction. The case $n=0$ is trivial. Suppose the statement holds for $n-1 \in \mathbb{N}$, and let us prove it for $n$. We have

$$
\begin{aligned}
(x \ominus n)+n & =((x \ominus(n-1)) \ominus 1)+n & & \text { (ind. def. of }-\ominus n) \\
& =((x \ominus(n-1)) \ominus 1)+1+(n-1) & & \\
& =((x \ominus(n-1)) \vee 1)+(n-1) & & \text { (Axiom P3) } \\
& =((x \ominus(n-1))+(n-1)) \vee n & & \text { (+ distr. over } \vee) \\
& =x \vee(n-1) \vee n & & \text { (ind. hyp.) } \\
& =x \vee n . & &
\end{aligned}
$$

Proposition 4.15. For every positive-unital commutative $\ell$-monoid $M$, the function $\eta_{M}^{0}: M \rightarrow(\mathrm{T}(M))^{+}$is bijective.

Proof. First, we prove that $\eta_{M}^{0}$ is injective. Let $x, y \in M$, and suppose $\eta_{M}^{0}(x)=$ $[x, 0]=[y, 0]=\eta_{M}^{0}(y)$. Then, $x+0=y+0$, and so $x=y$. Second, we prove that $\eta_{M}^{0}$ is surjective. Let $[x, n] \in(\mathbb{T}(M))^{+}$. Then,

$$
\begin{aligned}
{[x, n] } & =[x, n] \vee[0,0] \\
& =[x \vee n, n] \\
& =[(x \ominus n)+n, n] \\
& =[x \ominus n, 0] \\
& =\eta_{M}^{0}(x \ominus n) .
\end{aligned}
$$


We recall that two functors $F: \mathrm{A} \rightarrow \mathrm{B}$ and $G: \mathrm{B} \rightarrow \mathrm{A}$ are called quasiinverses if the functors $G F: \mathrm{A} \rightarrow \mathrm{A}$ and $F G: \mathrm{B} \rightarrow \mathrm{B}$ are naturally isomorphic to the identity functors on $A$ and $B$ respectively. Two categories $A$ and $B$ are equivalent if, and only if, there exist two quasi-inverses $F: \mathrm{A} \rightarrow \mathrm{B}$ and $G: \mathrm{B} \rightarrow \mathrm{A}[12$, Chapter IV, Section 4].

Theorem 4.16. The functors $(-)^{+}: \mathrm{u} \ell \mathrm{M} \rightarrow \mathrm{u} \ell \mathrm{M}^{+}$and $\mathrm{T}: \mathrm{u} \ell \mathrm{M}^{+} \rightarrow \mathrm{u} \ell \mathrm{M}$ are quasi-inverses.

Proof. The functors $1_{\mathrm{u} \ell \mathrm{M}^{+}}: \mathrm{u} \ell \mathrm{M}^{+} \rightarrow \mathrm{u} \ell \mathrm{M}^{+}$and $(-)^{+} \mathrm{T}: \mathrm{u} \ell \mathrm{M}^{+} \rightarrow \mathrm{u} \ell \mathrm{M}^{+}$are naturally isomorphic by Propositions $4.11,4.12$ and 4.15 . The functors $1_{\mathrm{u} \ell \mathrm{M}}$ : $\mathrm{u} \ell \mathrm{M} \rightarrow \mathrm{u} \ell \mathrm{M}$ and $\mathrm{T}(-)^{+}: \mathrm{u} \ell \mathrm{M} \rightarrow \mathrm{u} \ell \mathrm{M}$ are naturally isomorphic by Propositions 4.8 to 4.10 .

\section{Good sequences}

Definition 5.1. Let $A$ be an MV-monoidal algebra. A good pair in $A$ is a pair $\left(x_{0}, x_{1}\right)$ of elements of $A$ such that $x_{0} \oplus x_{1}=x_{0}$ and $x_{0} \odot x_{1}=x_{1}$. A good sequence in $A$ is a sequence $\left(x_{0}, x_{1}, x_{2}, \ldots\right)$ of elements of $A$ which is eventually 0 and such that, for each $n \in \mathbb{N},\left(x_{n}, x_{n+1}\right)$ is a good pair.

Instead of $\left(x_{0}, \ldots, x_{n}, 0,0,0, \ldots\right)$ we shall often write, more concisely, $\left(x_{0}, \ldots, x_{n}\right)$. Thus, if $0^{m}$ denotes an $m$-tuple of zeros, the good sequences $\left(x_{1}, \ldots, x_{n}\right)$ and $\left(x_{0}, \ldots, x_{n}, 0^{m}\right)$ are identical. For each $x \in A$, the sequence $(x, 0,0,0, \ldots)$ (which is always good) will be denoted by $(x)$.

Remark 5.2. In our definition of good pair we included both the condition $x_{0} \oplus x_{1}=x_{0}$ and the condition $x_{0} \odot x_{1}=x_{1}$ because, in general, they are not equivalent. As an example, one can take the MV-monoidal algebra consisting of three elements $\{0, a, 1\}$, where $a \oplus a=a$, and $a \odot a=0$.

To prove the equivalence between the categories of MV-algebras and unital Abelian $\ell$-groups (see [16] or [7]), Mundici used the facts that subdirectly irreducible MV-algebras are totally ordered and that good sequences in totally ordered MV-algebras are of the form $(1, \ldots, 1, x, 0,0, \ldots)$.

In this paper we do not make use of the Subdirect Representation Theorem (in fact, we do not make use of the axiom of choice) to establish the equivalence between $\mathrm{u} \ell \mathrm{M}$ and MVM. The reason why this is done is that, initially, the author was unable to prove that, in subdirectly irreducible MV-monoidal algebras, good sequences are of the form $(1, \ldots, 1, x, 0,0, \ldots)$. Eventually, such a proof was found, and the result is given in Corollary C.6. However, the result is not used in the present paper, for the following reasons. First, in this way, the proof that we provide for the equivalence between $\mathrm{u} \ell \mathrm{M}$ and MVM may be applied in similar settings, where the structure of subdirectly irreducible algebras is not known. Secondly, the proof we give does not rely on the axiom of choice. In particular, up to proving without the axiom of choice that the axioms of MV-monoidal algebras hold in any MV-algebra, we obtain a proof of the equivalence between unital Abelian $\ell$-groups and MV-algebras that does not make use of the axiom of choice. 
For a proof of our main result that does take advantage of the Subdirect Representation Theorem, the reader is invited to consult the author's Ph.D. thesis [2, Chapter 4].

\section{Basic properties of MV-monoidal algebras}

Remark 6.1. Inspection of the axioms that define MV-monoidal algebras shows that, for each MV-monoidal algebra $\langle A ; \oplus, \odot, \vee, \wedge, 0,1\rangle$, the 'dual' algebra $\langle A ; \odot, \oplus, \wedge, \vee, 1,0\rangle$ is also an MV-monoidal algebra.

We give a name to the right- and left-hand terms of Axioms A4 and A5; we will then prove that their interpretations in an MV-monoidal algebra coincide.

Notation 6.2. We set

$$
\begin{aligned}
& \sigma_{1}(x, y, z):=(x \oplus y) \odot((x \odot y) \oplus z) ; \\
& \sigma_{2}(x, y, z):=(x \odot y) \oplus((x \oplus y) \odot z) ; \\
& \sigma_{3}(x, y, z):=(x \odot(y \oplus z)) \oplus(y \odot z) ; \\
& \sigma_{4}(x, y, z):=(x \oplus(y \odot z)) \odot(y \oplus z) .
\end{aligned}
$$

Note that Axiom A5 can be written as $\sigma_{1}(x, y, z)=\sigma_{3}(x, y, z)$, Axiom A6 can be written as $\sigma_{2}(x, y, z)=\sigma_{4}(x, y, z)$, Axiom A6 can be written as $(x \odot y) \oplus z=\sigma_{1}(x, y, z) \vee z$, and Axiom A7 can be written as $(x \oplus y) \odot z=$ $\sigma_{2}(x, y, z) \wedge z$.

Lemma 6.3. Let $A$ be an $M V$-monoidal algebra. For all $i, j \in\{1,2,3,4\}$, every permutation $\rho:\{1,2,3\} \rightarrow\{1,2,3\}$ and all $x_{1}, x_{2}, x_{3} \in A$ we have

$$
\sigma_{i}\left(x_{1}, x_{2}, x_{3}\right)=\sigma_{j}\left(x_{\rho(1)}, x_{\rho(2)}, x_{\rho(3)}\right) .
$$

In other words, the terms $\sigma_{1}, \sigma_{2}, \sigma_{3}, \sigma_{4}$ in the theory of $M V$-monoidal algebras are all invariant under permutations of variables, and they coincide.

Proof. By commutativity of $\oplus$ and $\odot$, in the theory of MV-monoidal algebras $\sigma_{1}$ is invariant under transposition of the first and the second variables, and $\sigma_{3}$ is invariant under transposition of the second and the third ones. Moreover, by Axiom A4, we have $\sigma_{1}(x, y, z)=\sigma_{3}(x, y, z)$. Since any two distinct transpositions in the symmetric group on three elements generate the whole group, it follows that $\sigma_{1}$ and $\sigma_{3}$ are invariant under any permutation of the variables. By commutativity of $\oplus$ and $\odot$, we have $\sigma_{1}(x, y, z)=\sigma_{4}(z, y, x)$, and, by Axiom A5, we have $\sigma_{2}(x, y, z)=\sigma_{4}(x, y, z)$. We conclude that $\sigma_{1}, \sigma_{2}, \sigma_{3}$, $\sigma_{4}$ are invariant under permutations of variables, and they coincide.

In particular, Lemma 6.3 guarantees that we have

$$
\sigma_{1}(x, y, z)=\sigma_{2}(x, y, z)=\sigma_{3}(x, y, z)=\sigma_{4}(x, y, z) .
$$

Notation 6.4. For $x, y, z$ in an MV-monoidal algebra, we let $\sigma(x, y, z)$ denote the common value of $\sigma_{1}(x, y, z), \sigma_{2}(x, y, z), \sigma_{3}(x, y, z)$ and $\sigma_{4}(x, y, z)$. 
Lemma 6.5. For every $x$ in an $M V$-monoidal algebra we have $0 \leqslant x \leqslant 1$.

Proof. We have

$$
\begin{aligned}
x & =(x \odot 1) \oplus 0 \\
& =\sigma_{1}(x, 1,0) \vee 0 \\
& =\sigma_{3}(x, 1,0) \vee 0 \\
& =((x \odot(1 \oplus 0)) \oplus(1 \odot 0)) \vee 0 \\
& =((x \odot 1) \oplus 0) \vee 0 \\
& =x \vee 0 .
\end{aligned}
$$

Thus, $0 \leqslant x$. Dually, $x \leqslant 1$.

Lemma 6.6. For every $x$ in an $M V$-monoidal algebra, we have $x \oplus 1=1$ and $x \odot 0=0$.

Proof. We have

$$
\begin{aligned}
0 & =1 \odot 0 \\
& =(1 \vee x) \odot 0 \\
& =(1 \odot 0) \vee(x \odot 0) \\
& =0 \vee(x \odot 0) \\
& =x \odot 0 .
\end{aligned}
$$

Dually, $x \oplus 1=1$.

Lemma 6.7. The following properties hold for all $x, y, z, x^{\prime}, y^{\prime}$ in an $M V$-monoidal algebra.

(1) If $x \leqslant x^{\prime}$ and $y \leqslant y^{\prime}$, then $x \oplus y \leqslant x^{\prime} \oplus y^{\prime}$.

(2) If $x \leqslant x^{\prime}$ and $y \leqslant y^{\prime}$, then $x \odot y \leqslant x^{\prime} \odot y^{\prime}$.

(3) $x \leqslant y \Rightarrow x \oplus z \leqslant y \oplus z$

(4) $x \leqslant y \Rightarrow x \odot z \leqslant y \odot z$.

(5) $x \leqslant x \oplus y$.

(6) $x \geqslant x \odot y$.

Proof. Let us call $A$ the MV-monoidal algebra of the statement. Item (1) is guaranteed by the application of Lemma 3.1 to the commutative $\ell$-monoid $\langle A ; \oplus, \vee, \wedge, 0\rangle$. Item (2) is dual to item (1). Item (3) holds by item (1) together with the fact that $z \leqslant z$. Item (4) is dual to item (3). From $0 \leqslant y$ we obtain, by item (3), $x \oplus 0 \leqslant x \oplus y$. By Lemma 6.6, we have $x \oplus 0=x$. Therefore, $x \leqslant x \oplus y$, and so item (5) is proved. Item (6) is dual to item (5).

Lemma 6.8. For all $x, y, z$ in an MV-monoidal algebra we have

$$
x \odot(y \oplus z) \leqslant(x \odot y) \oplus z .
$$

Proof. Using Axioms A4-A7, we obtain

$$
x \odot(y \oplus z)=x \wedge \sigma(x, y, z) \leqslant \sigma(x, y, z) \leqslant \sigma(x, y, z) \vee z=(x \odot y) \oplus z .
$$


Lemma 6.9. Let $A$ be an $M V$-monoidal algebra, let $\left(x_{0}, x_{1}\right)$ be a good pair in $A$, and let $y \in A$. Then

$$
x_{0} \odot\left(x_{1} \oplus y\right)=x_{1} \oplus\left(x_{0} \odot y\right),
$$

and both these elements coincide with $\sigma\left(x_{0}, x_{1}, y\right)$.

Proof. We have

$$
\begin{aligned}
x_{0} \odot\left(x_{1} \oplus y\right) & =\left(x_{0} \oplus x_{1}\right) \odot\left(\left(x_{0} \odot x_{1}\right) \oplus y\right) & & \left(\left(x_{0}, x_{1}\right) \text { is good }\right) \\
& =\sigma_{1}\left(x_{0}, x_{1}, y\right) & & \left(\text { def. of } \sigma_{1}\right) \\
& =\sigma_{2}\left(x_{0}, x_{1}, y\right) & & (\text { Lemma } 6.3) \\
& =\left(x_{0} \odot x_{1}\right) \oplus\left(\left(x_{0} \oplus x_{1}\right) \odot y\right) & & \left(\text { def. of } \sigma_{2}\right) \\
& =x_{1} \oplus\left(x_{0} \odot y\right) . & & \left(\left(x_{0}, x_{1}\right) \text { is good }\right)
\end{aligned}
$$

Lemma 6.10. For all $x$ and $y$ in an $M V$-monoidal algebra, the pair $(x \oplus y, x \odot y)$ is good.

Proof. We have

$$
\begin{aligned}
(x \oplus y) \oplus(x \odot y) & =(1 \odot(x \oplus y)) \oplus(x \odot y) & & (\text { Axiom A2) } \\
& =\sigma_{3}(1, x, y) & & \left(\text { def. of } \sigma_{3}\right) \\
& =\sigma_{4}(1, x, y) & & (\text { Lemma 6.3) } \\
& =(1 \oplus(x \odot y)) \odot(x \oplus y) & & \left(\text { def. of } \sigma_{4}\right) \\
& =1 \odot(x \oplus y) & & (\text { Lemma 6.6) } \\
& =x \oplus y . & & (\text { Axiom A2) }
\end{aligned}
$$

Dually, $(x \oplus y) \odot(x \odot y)=x \odot y$.

\section{Operations on the set of good sequences}

We denote with $\mathbb{G}(A)$ the set of good sequences in an MV-monoidal algebra $A$. (In fact, $\mathbb{G}$ stands for 'good'.) We will endow $\mathbb{G}(A)$ with the structure of a positive-unital commutative $\ell$-monoid. We let $\mathbf{0}$ denote the good sequence $(0,0,0, \ldots)$, and we let 1 denote the good sequence $(1,0,0,0, \ldots)$. For good sequences $\mathbf{a}=\left(a_{0}, a_{1}, a_{2}, \ldots\right)$ and $\mathbf{b}=\left(b_{0}, b_{1}, b_{2}, \ldots\right)$, we set

$$
\mathbf{a} \vee \mathbf{b}:=\left(a_{0} \vee b_{0}, a_{1} \vee b_{1}, a_{2} \vee b_{2}, \ldots\right),
$$

and

$$
\mathbf{a} \wedge \mathbf{b}:=\left(a_{0} \wedge b_{0}, a_{1} \wedge b_{1}, a_{2} \wedge b_{2}, \ldots\right) .
$$

Proposition 7.2 below asserts that $\mathbf{a} \vee \mathbf{b}$ and $\mathbf{a} \wedge \mathbf{b}$ are good sequences. To prove it, we establish the following lemmas.

Lemma 7.1. For all good pairs $\left(x_{0}, x_{1}\right)$ and $\left(y_{0}, y_{1}\right)$ in an $M V$-monoidal algebra, the pairs $\left(x_{0} \vee y_{0}, x_{1} \vee y_{1}\right)$ and $\left(x_{0} \wedge y_{0}, x_{1} \wedge y_{1}\right)$ are good. 
Proof. We prove that $\left(x_{0} \vee y_{0}, x_{1} \vee y_{1}\right)$ is a good pair. We have

$$
\begin{aligned}
\left(x_{0}\right. & \left.\vee y_{0}\right) \oplus\left(x_{1} \vee y_{1}\right) \\
& =\left(x_{0} \oplus x_{1}\right) \vee\left(x_{0} \oplus y_{1}\right) \vee\left(y_{0} \oplus x_{1}\right) \vee\left(y_{0} \oplus y_{1}\right) \\
& =x_{0} \vee\left(x_{0} \oplus y_{1}\right) \vee\left(y_{0} \oplus x_{1}\right) \vee y_{0} \\
& =\left(x_{0} \oplus y_{1}\right) \vee\left(y_{0} \oplus x_{1}\right) \\
& =\left(x_{0} \oplus\left(y_{0} \odot y_{1}\right)\right) \vee\left(y_{0} \oplus\left(x_{0} \odot x_{1}\right)\right) \\
& =x_{0} \vee \sigma\left(x_{0}, y_{0}, y_{1}\right) \vee y_{0} \vee \sigma\left(x_{0}, x_{1}, y_{0}\right) \\
& =x_{0} \vee\left(\left(x_{0} \oplus y_{1}\right) \odot y_{0}\right) \vee y_{0} \vee\left(x_{0} \odot\left(x_{1} \oplus y_{0}\right)\right) \\
& =\left(x_{0} \vee\left(x_{0} \odot\left(x_{1} \oplus y_{0}\right)\right)\right) \vee\left(\left(\left(x_{0} \oplus y_{1}\right) \odot y_{0}\right) \vee y_{0}\right) \\
& =x_{0} \vee y_{0} .
\end{aligned}
$$$$
(\oplus \text { distr. over } \vee \text { ) }
$$$$
\left(\left(x_{0}, x_{1}\right),\left(y_{0}, y_{1}\right) \operatorname{good}\right)
$$$$
\text { (Lemma 6.7.((5))) }
$$$$
\left(\left(x_{0}, x_{1}\right),\left(y_{0}, y_{1}\right) \text { good }\right)
$$$$
\text { (Axiom A6) }
$$$$
\text { (Lemma 6.7.((6))) }
$$

Moreover, we have

$$
\begin{aligned}
\left(x_{0}\right. & \left.\vee y_{0}\right) \odot\left(x_{1} \vee y_{1}\right) & & \\
& =\left(x_{0} \odot x_{1}\right) \vee\left(x_{0} \odot y_{1}\right) \vee\left(y_{0} \odot x_{1}\right) \vee\left(y_{0} \odot y_{1}\right) & & (\oplus \text { distr. over } \vee) \\
& =x_{1} \vee\left(x_{0} \odot y_{1}\right) \vee\left(y_{0} \odot x_{1}\right) \vee y_{1} & & \left(\left(x_{0}, x_{1}\right),\left(y_{0}, y_{1}\right)\right. \\
& =x_{1} \vee y_{1} . & & (\text { Lemma 6.7. }((6)))
\end{aligned}
$$$$
\left(\left(x_{0}, x_{1}\right),\left(y_{0}, y_{1}\right) \text { good }\right)
$$$$
\text { (Lemma 6.7.((6))) }
$$

Hence, $\left(x_{0} \vee y_{0}, x_{1} \vee y_{1}\right)$ is good. Dually, $\left(x_{0} \wedge y_{0}, x_{1} \wedge y_{1}\right)$ is good.

Proposition 7.2. For all good sequences $\mathbf{a}$ and $\mathbf{b}$ in an $M V$-monoidal algebra, the sequences $\mathbf{a} \vee \mathbf{b}$ and $\mathbf{a} \wedge \mathbf{b}$ are good.

Proof. By Lemma 7.1.

Proposition 7.3. Let $A$ be an $M V$-monoidal algebra. Then, $\langle\mathbb{G}(A) ; \vee, \wedge\rangle$ is a distributive lattice.

Proof. $\langle\mathbb{G}(A) ; \vee, \wedge\rangle$ is a distributive lattice, because $\vee$ and $\wedge$ are applied componentwise, and $\langle A ; \vee, \wedge\rangle$ is a distributive lattice.

For $A$ an MV-monoidal algebra, we have a partial order $\leqslant$ on $\mathbb{G}(A)$, induced by the lattice operations. Since the lattice operations are defined componentwise, we have the following.

Remark 7.4. Let $A$ be an MV-monoidal algebra. For all good sequences $\mathbf{a}=$ $\left(a_{0}, a_{1}, a_{2}, \ldots\right)$ and $\mathbf{b}=\left(b_{0}, b_{1}, b_{2}, \ldots\right)$ in $A$, we have $\mathbf{a} \leqslant \mathbf{b}$ if, and only if, for all $n \in \mathbb{N}, a_{n} \leqslant b_{n}$.

Now we want to define the sum of good sequences. Given two good sequences $\mathbf{a}=\left(a_{0}, a_{1}, a_{2}, \ldots\right)$ and $\mathbf{b}=\left(b_{0}, b_{1}, b_{2}, \ldots\right)$ in an MV-monoidal algebra, there are two natural ways to define a sequence $\mathbf{c}=\left(c_{0}, c_{1}, c_{2}, \ldots\right)$ as the sum of $\mathbf{a}$ and $\mathbf{b}$. The first one is

$$
c_{n}:=\left(a_{0} \oplus b_{n}\right) \odot\left(a_{1} \oplus b_{n-1}\right) \odot \cdots \odot\left(a_{n-1} \oplus b_{1}\right) \odot\left(a_{n} \oplus b_{0}\right),
$$

and the second one is $c_{n}:=b_{n} \oplus\left(a_{0} \odot b_{n-1}\right) \oplus\left(a_{1} \odot b_{n-2}\right) \oplus \cdots \oplus\left(a_{n-2} \odot b_{1}\right) \oplus\left(a_{n-1} \odot b_{0}\right) \oplus a_{n}$. Our first aim, reached in Lemma 7.8 below, is to show that these two ways coincide. 
Lemma 7.5. Let $x_{0}, \ldots, x_{n}, y_{0}, \ldots, y_{m}$ be elements of an $M V$-monoidal algebra and suppose that, for every $i \in\{0, \ldots, n\}$ and every $j \in\{0, \ldots, m\}$, the pair $\left(x_{i}, y_{j}\right)$ is good. Then, the pair

$$
\left(x_{0} \odot \cdots \odot x_{n}, y_{0} \oplus \cdots \oplus y_{m}\right)
$$

is good.

Proof. The statement is trivial for $(n, m)=(0,0)$. The statement is true for $(n, m)=(1,0)$ because

$$
\left(x_{0} \odot x_{1}\right) \oplus y_{0} \stackrel{\text { Lemma }}{=}{ }^{6.9} x_{0} \odot\left(x_{1} \oplus y_{0}\right)=x_{0} \odot x_{1},
$$

and

$$
\left(x_{0} \odot x_{1}\right) \odot y_{0}=x_{0} \odot\left(x_{1} \odot y_{0}\right)=x_{0} \odot y_{0}=y_{0} .
$$

The case $(n, m)=(0,1)$ is analogous. Let $(n, m) \in \mathbb{N} \times \mathbb{N} \backslash\{(0,0),(0,1),(1,0)\}$, and suppose that the statement is true for each $(h, k) \in \mathbb{N} \times \mathbb{N}$ such that $(h, k) \neq$ $(n, m), h \leqslant n$ and $k \leqslant m$. We prove that the statement holds for $(n, m)$. At least one of the two conditions $n \neq 0$ and $m \neq 0$ holds. Suppose, for example, $n \neq 0$. Then, by inductive hypothesis, the pairs $\left(x_{0} \odot \cdots \odot x_{n-1}, y_{0} \oplus \cdots \oplus y_{m}\right)$ and $\left(x_{n}, y_{0} \oplus \cdots \oplus y_{m}\right)$ are good. Now we apply the statement for the case $(1,0)$, and we obtain that $\left(x_{0} \odot \cdots \odot x_{n}, y_{0} \oplus \cdots \oplus y_{m}\right)$ is a good pair. The case $m \neq 0$ is analogous.

Lemma 7.6. Let $A$ be an $M V$-monoidal algebra. For every good pair $(x, y)$ in $A$ and every $u \in A$, the pairs $(x \oplus u, y)$ and $(x, y \odot u)$ are good.

Proof. The pair $(x \oplus u, y)$ is good because we have $(x \oplus u) \oplus y=(x \oplus y) \oplus u=$ $x \oplus u$, and, by Lemma 6.7, we have $y=x \odot y \leqslant(x \oplus u) \odot y \leqslant y$, which implies $(x \oplus u) \odot y=y$. Dually, $(x, y \odot u)$ is good.

Lemma 7.7. If $(x, y)$ and $(y, z)$ are good pairs in an $M V$-monoidal algebra, then $(x, z)$ is a good pair.

Proof. We have

and

$$
\begin{aligned}
& x \oplus z=(x \oplus y) \oplus z=x \oplus(y \oplus z)=x \oplus y=x, \\
& x \odot z=x \odot(y \odot z)=(x \odot y) \odot z=y \odot z=z .
\end{aligned}
$$

Lemma 7.8. Let $A$ be an $M V$-monoidal algebra, let $m \in \mathbb{N}$ and let $\left(a_{0}, \ldots, a_{m}\right)$ and $\left(b_{0}, \ldots, b_{m}\right)$ be good sequences in $A$. Then

$$
\left(a_{0} \odot b_{m}\right) \oplus \cdots \oplus\left(a_{m} \odot b_{0}\right)=a_{0} \odot\left(a_{1} \oplus b_{m}\right) \odot \cdots \odot\left(a_{m} \oplus b_{1}\right) \odot b_{0},
$$

and

$$
\left(a_{0} \oplus b_{m}\right) \odot \cdots \odot\left(a_{m} \oplus b_{0}\right)=b_{m} \oplus\left(a_{0} \odot b_{m-1}\right) \oplus \cdots \oplus\left(a_{m-1} \odot b_{0}\right) \oplus a_{m} .
$$


Proof. We prove the first equality by induction on $m \in \mathbb{N}$. The case $m=0$ is trivial. Let $m \in \mathbb{N} \backslash\{0\}$ and suppose that the statement holds for $n-1$. By the inductive hypothesis, we have

$$
\left(a_{0} \odot b_{m}\right) \oplus \cdots \oplus\left(a_{m} \odot b_{0}\right)=\left(a_{0} \odot\left(a_{1} \oplus b_{m}\right) \odot \cdots \odot\left(a_{m-1} \oplus b_{2}\right) \odot b_{1}\right) \oplus\left(a_{m} \odot b_{0}\right) .
$$

By Lemma 7.6, the pair $\left(b_{0}, a_{0} \odot\left(a_{1} \oplus b_{m}\right) \odot \cdots \odot\left(a_{m-1} \oplus b_{2}\right) \odot b_{1}\right)$ is good. Therefore, by Lemma 6.9, we have

$$
\begin{aligned}
& \left(a_{0} \odot\left(a_{1} \oplus b_{m}\right) \odot \cdots \odot\left(a_{m-1} \oplus b_{2}\right) \odot b_{1}\right) \oplus\left(a_{m} \odot b_{0}\right) \\
& \quad=\left(\left(a_{0} \odot\left(a_{1} \oplus b_{m}\right) \odot \cdots \odot\left(a_{m-1} \oplus b_{2}\right) \odot b_{1}\right) \oplus a_{m}\right) \odot b_{0} .
\end{aligned}
$$

By Lemma 7.7 , the pairs $\left(a_{0}, a_{m}\right),\left(a_{1}, a_{m}\right), \ldots\left(a_{m-2}, a_{m}\right),\left(a_{m-1}, a_{m}\right)$ are good. By Lemma 7.6, the pairs $\left(a_{0}, a_{m}\right),\left(a_{1} \oplus b_{m}, a_{m}\right), \ldots,\left(a_{m-2} \oplus b_{3}, a_{m}\right)$, $\left(a_{m-1} \oplus b_{2}, a_{m}\right)$ are good. By Lemma 7.5, the pair

$$
\left(a_{0} \odot\left(a_{1} \oplus b_{m}\right) \odot \cdots \odot\left(a_{m-1} \oplus b_{2}\right), a_{m}\right)
$$

is good. Thus, by Lemma 6.9, we have

$$
\begin{aligned}
& \left(\left(a_{0} \odot\left(a_{1} \oplus b_{m}\right) \odot \cdots \odot\left(a_{m-1} \oplus b_{2}\right) \odot b_{1}\right) \oplus a_{m}\right) \odot b_{0} \\
& \quad=\left(\left(a_{0} \odot\left(a_{1} \oplus b_{m}\right) \odot \cdots \odot\left(a_{m-1} \oplus b_{2}\right)\right) \odot\left(b_{1} \oplus a_{m}\right)\right) \odot b_{0} .
\end{aligned}
$$

The chain of equalities established in Eqs. (7.1)-(7.3) settles the first equality of the statement. The second one is dual.

Given two good sequences $\mathbf{a}=\left(a_{0}, a_{1}, a_{2}, \ldots\right)$ and $\mathbf{b}=\left(b_{0}, b_{1}, b_{2}, \ldots\right)$ in an MV-monoidal algebra, we set

$$
\mathbf{a}+\mathbf{b}:=\left(c_{0}, c_{1}, c_{2}, \ldots\right),
$$

where

$$
c_{n}:=\left(a_{0} \oplus b_{n}\right) \odot\left(a_{1} \oplus b_{n-1}\right) \odot \cdots \odot\left(a_{n-1} \oplus b_{1}\right) \odot\left(a_{n} \oplus b_{0}\right),
$$

or, equivalently (by Lemma 7.8),

$$
c_{n}:=b_{n} \oplus\left(a_{0} \odot b_{n-1}\right) \oplus\left(a_{1} \odot b_{n-2}\right) \oplus \cdots \oplus\left(a_{n-2} \odot b_{1}\right) \oplus\left(a_{n-1} \odot b_{0}\right) \oplus a_{n} .
$$

In Proposition 7.10 below, we show that $\mathbf{a}+\mathbf{b}$ is a good sequence. In preparation for it, we establish the following lemma.

Lemma 7.9. Let $\left(a_{0}, \ldots, a_{n}\right)$ and $\left(b_{0}, \ldots, b_{n}\right)$ be good sequences in an $M V$ monoidal algebra. Then, the pair

$$
\left(\left(a_{0} \oplus b_{n}\right) \odot \cdots \odot\left(a_{n} \oplus b_{0}\right),\left(a_{0} \odot b_{n}\right) \oplus \cdots \oplus\left(a_{n} \odot b_{0}\right)\right)
$$

is good.

Proof. By Lemma 7.5, it is enough to show that, for $i, j \in\{0, \ldots, m\}$, the pair $\left(a_{i} \oplus b_{m-i}, a_{j} \odot b_{m-j}\right)$ is good. The case $i=j$ is covered by Lemma 6.10. If $i<j$, then, by Lemma 7.7, the pair $\left(a_{i}, a_{j}\right)$ is good; by Lemma 7.6, the pair $\left(a_{i} \oplus b_{m-i}, a_{j} \odot b_{m-j}\right)$ is good. If $i>j$, then, by Lemma 7.7, the pair $\left(b_{m-i}, b_{m-j}\right)$ is good; by Lemma 7.6 , the pair $\left(a_{i} \oplus b_{m-i}, a_{j} \odot b_{m-j}\right)$ is good. 
Proposition 7.10. For all good sequences $\mathbf{a}$ and $\mathbf{b}$ in an $M V$-monoidal algebra, the sequence $\mathbf{a}+\mathbf{b}$ is good.

Proof. Let $\mathbf{a}=\left(a_{0}, \ldots, a_{m}\right)$ and $\mathbf{b}=\left(b_{0}, \ldots, b_{m}\right)$. Set $\mathbf{c}:=\mathbf{a}+\mathbf{b}$, and write $\mathbf{c}=\left(c_{0}, c_{1}, c_{2}, \ldots\right)$. Then, for $j>2 m$ we have $c_{j}=0$. For all $n \in \mathbb{N}$ we have

$$
\begin{aligned}
c_{n} & =\left(a_{0} \oplus b_{n}\right) \odot \cdots \odot\left(a_{n} \oplus b_{0}\right) \\
& =\left(1 \oplus b_{n+1}\right) \odot\left(a_{0} \oplus b_{n}\right) \odot \cdots \odot\left(a_{n} \oplus b_{0}\right) \odot\left(a_{n+1} \oplus 1\right),
\end{aligned}
$$

and

$$
c_{n+1}=\left(1 \odot b_{n+1}\right) \oplus\left(a_{0} \odot b_{n}\right) \oplus \cdots \oplus\left(a_{n} \odot b_{0}\right) \oplus\left(a_{n+1} \odot 1\right) .
$$

By Lemma 7.9 , the pair $\left(c_{n}, c_{n+1}\right)$ is good.

Proposition 7.11. Addition of good sequences is commutative.

Proof. By commutativity of $\oplus$ and $\odot$, we have

$$
\mathbf{a}+\mathbf{b}=\left(a_{0} \oplus b_{n}\right) \odot \cdots \odot\left(a_{n} \oplus b_{0}\right)=\left(b_{0} \oplus a_{n}\right) \odot \cdots \odot\left(b_{n} \oplus a_{0}\right)=\mathbf{b}+\mathbf{a} .
$$

Remark 7.12. Let $A$ be an MV-monoidal algebra, and let $\mathbf{a} \in \mathbb{G}(A)$. Then, $\mathbf{a}+\mathbf{0}=\mathbf{a}$.

Now, we show that, for all good sequences $\mathbf{x}, \mathbf{y}, \mathbf{z}$ in an MV-monoidal algebra, we have $(\mathbf{x}+\mathbf{y})+\mathbf{z}=\mathbf{x}+(\mathbf{y}+\mathbf{z})$. A direct verification, which seems difficult in general, becomes treatable when $\mathbf{y}$ is of the form $\left(y_{0}, 0,0, \ldots\right)$. Light's associativity test guarantees that this is enough to imply associativity, thanks to the fact that the elements of the form $\left(y_{0}, 0,0, \ldots\right)$ generate $\mathbb{G}(A)$. In the following, we carry out the details.

Lemma 7.13. For every good sequence $\left(a_{0}, \ldots, a_{n}\right)$ in an $M V$-monoidal algebra we have

$$
\left(a_{0}, \ldots, a_{n}\right)=\left(a_{0}, \ldots, a_{n-1}\right)+\left(a_{n}\right) .
$$

Proof. Set $\left(b_{0}, b_{1}, b_{2}, \ldots\right):=\left(a_{0}, \ldots, a_{n-1}\right)+\left(a_{n}\right)$. For $k \in\{0, \ldots, n-1\}$, we have

$$
b_{k}=a_{0} \odot a_{1} \odot \cdots \odot a_{k-1} \odot\left(a_{k} \oplus a_{n}\right)=a_{0} \odot a_{1} \odot \cdots \odot a_{k-1} \odot a_{k}=a_{k} .
$$

Moreover, $b_{n}=a_{0} \odot a_{1} \odot \cdots \odot a_{n-1} \odot a_{n}=a_{n}$, and, for $k>n$, we have $b_{k}=0$. In conclusion, $\left(a_{0}, \ldots, a_{n}\right)=\left(b_{0}, b_{1}, b_{2}, \ldots\right)=\left(a_{0}, \ldots, a_{n-1}\right)+\left(a_{n}\right)$.

Notation 7.14. A magma $\langle X ; \cdot\rangle$ consists of a set $X$ and a binary operation . on $X$. Given a subset $T$ of a magma $X$, we define, inductively on $n \in \mathbb{N}_{>0}$, the subset $T_{n}$; we set $T_{1}:=T$, and, for $n \in \mathbb{N}_{>0}, T_{n}:=\left\{t z \mid t \in T, z \in T_{n-1}\right\} \cup\{z t \mid$ $\left.t \in T, z \in T_{n-1}\right\}$. Roughly speaking, $T_{n}$ is the set of elements of $X$ that can be obtained with at most $n$ occurrences of elements of $T$ via application of the operation $\cdot$. We say that $T$ generates $X$ if $\bigcup_{n \in \mathbb{N}_{>0}} T_{n}=X$.

Lemma 7.15. For every $M V$-monoidal algebra $A$, the set $\{(x) \in \mathbb{G}(A) \mid x \in$ $A\}$ generates the magma $\langle\mathbb{G}(A) ;+\rangle$.

Proof. By induction, using Lemma 7.13. 
Lemma 7.16. (Light's associativity test) Let $\langle X ; \cdot\rangle$ be a magma, and let $T$ be a subset of $X$ that generates $X$. Suppose that, for every $x, z \in X$ and $t \in T$, we have $(x t) z=x(t z)$. Then, the operation - is associative.

Proof. Since $T$ generates $X$, we have $\bigcup_{n \in \mathbb{N}_{>0}} T_{n}=X$. We prove, by induction on $n \in \mathbb{N}_{>0}$, that, for every $y \in T_{n}$, and every $x, y \in X$, we have $(x y) z=x(y z)$. The case $n=1$ is ensured by hypothesis. Let $n \geqslant 2$, and suppose that the cases $1, \ldots, n-1$ hold. Then, either $y=t y^{\prime}$ or $y=y^{\prime} t$, for some $t \in T$ and $y^{\prime} \in T_{n}$. Suppose, for example, $y=t y^{\prime}$. Then, we have

$$
(x y) z=\left(x\left(t y^{\prime}\right)\right) z=\left((x t) y^{\prime}\right) z=(x t)\left(y^{\prime} z\right)=x\left(t\left(y^{\prime} z\right)\right)=x\left(\left(t y^{\prime}\right) z\right)=x(y z) .
$$

The case $y=y^{\prime} t$ is analogous.

Lemma 7.17. Let $A$ be an $M V$-monoidal algebra, let $\left(a_{0}, a_{1}\right)$ and $\left(b_{0}, b_{1}\right)$ be good pairs in $A$, and let $x \in A$. Then

$$
\left(a_{0} \oplus\left(x \odot b_{0}\right) \oplus b_{1}\right) \odot\left(a_{1} \oplus x \oplus b_{0}\right)=\left(a_{0} \oplus x \oplus b_{1}\right) \odot\left(a_{1} \oplus\left(a_{0} \odot x\right) \oplus b_{0}\right),
$$

and both sides coincide with $a_{1} \oplus \sigma\left(a_{0}, x, b_{0}\right) \oplus b_{1}$.

Proof. Since the pair $\left(a_{0}, a_{1}\right)$ is good, it follows from Lemma 7.6 that the pair $\left(a_{0} \oplus\left(x \odot b_{0}\right) \oplus b_{1}, a_{1}\right)$ is good. Since the pair $\left(b_{0}, b_{1}\right)$ is good, it follows from Lemma 7.6 that the pair $\left(x \oplus b_{0}, b_{1}\right)$ is good. Therefore, we have

$$
\begin{aligned}
\left(a_{0}\right. & \left.\oplus\left(x \odot b_{0}\right) \oplus b_{1}\right) \odot\left(a_{1} \oplus x \oplus b_{0}\right) \\
& =a_{1} \oplus\left(\left(a_{0} \oplus\left(x \odot b_{0}\right) \oplus b_{1}\right) \odot\left(x \oplus b_{0}\right)\right) \\
& =a_{1} \oplus\left(\left(a_{0} \oplus\left(x \odot b_{0}\right)\right) \odot\left(x \oplus b_{0}\right)\right) \oplus b_{1} \\
& =a_{1} \oplus \sigma\left(a_{0}, x, b_{0}\right) \oplus b_{1} .
\end{aligned}
$$

Analogously, $\left(a_{0} \oplus x \oplus b_{1}\right) \odot\left(a_{1} \oplus\left(a_{0} \odot x\right) \oplus b_{0}\right)=a_{1} \oplus \sigma\left(a_{0}, x, b_{0}\right) \oplus b_{1}$.

Lemma 7.18. Let $A$ be an $M V$-monoidal algebra, let $n \in \mathbb{N}_{>0}$, let $\left(a_{0}, \ldots, a_{n}\right)$ and $\left(b_{0}, \ldots, b_{n}\right)$ be good sequences in $A$, and let $x \in A$. Then

$$
\bigodot_{i=0}^{n} a_{i} \oplus\left(x \odot b_{n-i-1}\right) \oplus b_{n-i}=\bigodot_{i=0}^{n} a_{i} \oplus\left(a_{i-1} \odot x\right) \oplus b_{n-i} .
$$

Proof. We prove the statement by induction on $n \in \mathbb{N}_{>0}$. The case $n=1$ is Lemma 7.17. Now let $n \in \mathbb{N} \backslash\{0,1\}$, and suppose that the statement holds for $n-1$. In the following chain of equalities, the second equality is obtained by an application of Lemma 7.17 with respect to the good pairs $\left(a_{n-1}, a_{n}\right)$ and $\left(b_{0}, b_{1}\right)$, and the third equality is obtained by an application of the inductive hypothesis with respect to the good sequences $\left(a_{0}, \ldots, a_{n-1}\right)$ and $\left(b_{1}, \ldots, b_{n}\right)$.

$$
\begin{aligned}
& \bigodot_{i=0}^{n} a_{i} \oplus\left(x \odot b_{n-i-1}\right) \oplus b_{n-i} \\
&=\left(\bigodot_{i=0}^{n-2} a_{i} \oplus\left(x \odot b_{n-i-1}\right) \oplus b_{n-i}\right) \odot\left(a_{n-1} \oplus\left(x \odot b_{0}\right) \oplus b_{1}\right) \\
& \odot\left(a_{n} \oplus x \oplus b_{1}\right)
\end{aligned}
$$




$$
\begin{aligned}
= & \left(\bigodot_{i=0}^{n-2} a_{i} \oplus\left(x \odot b_{n-i-1}\right) \oplus b_{n-i}\right) \odot\left(a_{n-1} \oplus x \oplus b_{1}\right) \\
& \odot\left(a_{n} \oplus\left(a_{n-1} \odot x\right) \oplus b_{1}\right) \\
= & \left(\bigodot_{i=0}^{n} a_{i} \oplus\left(a_{i-1} \odot x\right) \oplus b_{n-i}\right) \odot\left(a_{n} \oplus\left(a_{n-1} \odot x\right) \oplus b_{1}\right) \\
= & \bigodot_{i=0}^{n} a_{i} \oplus\left(a_{i-1} \odot x\right) \oplus b_{n-i} .
\end{aligned}
$$

Lemma 7.19. Let $A$ be an $M V$-monoidal algebra, let $\mathbf{a}$ and $\mathbf{b}$ be good sequences in $A$, and let $x \in A$. Then,

$$
(\mathbf{a}+(x))+\mathbf{b}=\mathbf{a}+((x)+\mathbf{b}) .
$$

Proof. Set $\mathbf{d}:=\mathbf{a}+(x)$ and $\mathbf{e}:=(x)+\mathbf{b}$. For every $n \in \mathbb{N}$, we have $d_{n}=a_{n} \oplus$ $\left(a_{n-1} \odot x\right)$ and $e_{n}=\left(x \odot b_{n-1}\right) \oplus b_{n}$. We set $\mathbf{f}:=(\mathbf{a}+(x))+\mathbf{b}$ and $\mathbf{g}:=\mathbf{a}+((x)+\mathbf{b})$. For every $n \in \mathbb{N}$, we have

$$
\begin{aligned}
f_{n} & =\bigodot_{i=0}^{n} d_{i} \oplus b_{n-i} \\
& =\bigodot_{i=0}^{n} a_{i} \oplus\left(a_{i-1} \odot x\right) \oplus b_{n-i} \\
& =\bigodot_{i=0}^{n} a_{i} \oplus\left(x \odot b_{n-i-1}\right) \oplus b_{n-i} \\
& =\bigodot_{i=0}^{n} a_{i} \oplus e_{n-i} \\
& =g_{n} .
\end{aligned}
$$

Proposition 7.20. Addition of good sequences is associative.

Proof. By Lemmas 7.15, 7.16 and 7.19.

Our next aim - reached in Proposition 7.23 below - is to show that good sequences satisfy $\mathbf{a}+(\mathbf{b} \vee \mathbf{c})=(\mathbf{a}+\mathbf{b}) \vee(\mathbf{a}+\mathbf{c})$. We need some lemmas.

Lemma 7.21. Let $A$ be an $M V$-monoidal algebra, let $\left(x_{0}, x_{1}\right)$ and $\left(y_{0}, y_{1}\right)$ be good pairs in $A$ and let $z \in A$. Then

$$
\left(\left(z \oplus x_{1}\right) \odot x_{0}\right) \vee\left(\left(z \oplus y_{1}\right) \odot y_{0}\right)=\left(z \oplus\left(x_{1} \vee y_{1}\right)\right) \odot\left(x_{0} \vee y_{0}\right)
$$

Proof. We have

$$
\begin{aligned}
(z & \left.\oplus\left(x_{1} \vee y_{1}\right)\right) \odot\left(x_{0} \vee y_{0}\right) \\
& =\left(\left(z \oplus x_{1}\right) \vee\left(z \oplus y_{1}\right)\right) \odot\left(x_{0} \vee y_{0}\right) \\
& =\left(\left(z \oplus x_{1}\right) \odot\left(x_{0} \vee y_{0}\right)\right) \vee\left(\left(z \oplus y_{1}\right) \odot\left(x_{0} \vee y_{0}\right)\right) \\
& =\left(\left(z \oplus x_{1}\right) \odot x_{0}\right) \vee\left(\left(z \oplus x_{1}\right) \odot y_{0}\right) \vee\left(\left(z \oplus y_{1}\right) \odot x_{0}\right) \vee\left(\left(z \oplus y_{1}\right) \odot y_{0}\right)
\end{aligned}
$$




$$
=\sigma\left(z, x_{1}, x_{0}\right) \vee\left(\left(z \oplus x_{1}\right) \odot y_{0}\right) \vee\left(\left(z \oplus y_{1}\right) \odot x_{0}\right) \vee \sigma\left(z, y_{1}, y_{0}\right),
$$

where the last equality follows from Lemma 6.9. We have

$$
\begin{aligned}
\left(z \oplus x_{1}\right) \odot y_{0} & =\left(z \oplus\left(x_{1} \odot x_{0}\right)\right) \odot y_{0} & & \left(\left(x_{0}, x_{1}\right) \text { is good }\right) \\
& =\left(z \vee \sigma\left(z, x_{1}, x_{0}\right)\right) \odot y_{0} & & \text { (Axiom A6) } \\
& =\left(z \odot y_{0}\right) \vee\left(\sigma\left(z, x_{1}, x_{0}\right) \odot y_{0}\right) & & (\odot \text { distr. over } \vee) \\
& \leqslant\left(\left(z \oplus y_{1}\right) \odot y_{0}\right) \vee \sigma\left(z, x_{1}, x_{0}\right) & & (\text { Lemma 6.7) } \\
& =\sigma\left(z, y_{1}, y_{0}\right) \vee \sigma\left(z, x_{1}, x_{0}\right) . & & (\text { Lemma 6.9) }
\end{aligned}
$$

Analogously, $\left(z \oplus y_{1}\right) \odot x_{0} \leqslant \sigma\left(z, x_{1}, x_{0}\right) \vee \sigma\left(z, y_{1}, y_{0}\right)$. Therefore, (7.4) equals $\sigma\left(z, x_{1}, x_{0}\right) \vee \sigma\left(z, y_{1}, y_{0}\right)$, i.e. $\left(\left(z \oplus x_{1}\right) \odot x_{0}\right) \vee\left(\left(z \oplus y_{1}\right) \odot y_{0}\right)$.

Lemma 7.22. Let $A$ be an $M V$-monoidal algebra, let $a \in A$ and let $\mathbf{b}$ and $\mathbf{c}$ be good sequences in $A$. Then, $(a)+(\mathbf{b} \vee \mathbf{c})=((a)+\mathbf{b}) \vee((a)+\mathbf{c})$.

Proof. We set $\mathbf{d}:=(a)+(\mathbf{b} \vee \mathbf{c})$. Then,

$$
\begin{aligned}
d_{n} & =\left(a \oplus\left(b_{n} \vee c_{n}\right)\right) \odot\left(b_{n-1} \vee c_{n-1}\right) \odot \cdots \odot\left(b_{0} \vee c_{0}\right) \\
& =\left(a \oplus\left(b_{n} \vee c_{n}\right)\right) \odot\left(b_{n-1} \vee c_{n-1}\right) .
\end{aligned}
$$

We set $\mathbf{f}:=(a)+\mathbf{b}, \mathbf{g}:=(a)+\mathbf{c}$ and $\mathbf{h}:=\mathbf{f} \vee \mathbf{g}=((a)+\mathbf{b}) \vee((a)+\mathbf{c})$. We have

$$
\begin{aligned}
& f_{n}=\left(a \oplus b_{n}\right) \odot b_{n-1} \odot \cdots \odot b_{0}=\left(a \oplus b_{n}\right) \odot b_{n-1}, \\
& g_{n}=\left(a \oplus c_{n}\right) \odot c_{n-1} \odot \cdots \odot c_{0}=\left(a \oplus c_{n}\right) \odot c_{n-1},
\end{aligned}
$$

and

$$
\begin{aligned}
h_{n} & =f_{n} \vee g_{n} \\
& =\left(\left(a \oplus b_{n}\right) \odot b_{n-1}\right) \vee\left(\left(a \oplus c_{n}\right) \odot c_{n-1}\right) \\
& =\left(a \oplus\left(b_{n} \vee c_{n}\right)\right) \odot\left(b_{n-1} \vee c_{n-1}\right) \\
& =d_{n} .
\end{aligned}
$$

Proposition 7.23. For all good sequences $\mathbf{a}, \mathbf{b}, \mathbf{c}$ in an $M V$-monoidal algebra, we have

$$
\mathbf{a}+(\mathbf{b} \vee \mathbf{c})=(\mathbf{a}+\mathbf{b}) \vee(\mathbf{a}+\mathbf{c})
$$

and

$$
\mathbf{a}+(\mathbf{b} \wedge \mathbf{c})=(\mathbf{a}+\mathbf{b}) \wedge(\mathbf{a}+\mathbf{c})
$$

Proof. Let us prove the first equality: the second one is analogous. Let $A$ be the MV-monoidal algebra of the statement. Set $\hat{A}:=\{(x) \in \mathbb{G}(A) \mid x \in A\}$. By Lemma 7.15, $\hat{A}$ generates the magma $\langle\mathbb{G}(A) ;+\rangle$. Following Notation 7.14 , for $n \in \mathbb{N}_{>0}$, we let $\hat{A}_{n}$ denote the set of elements of $\mathbb{G}(A)$ which can be obtained with at most $n$ occurrences of elements of $\hat{A}$ via application of + . We prove by induction on $n \in \mathbb{N}_{>0}$ that, for all $\mathbf{a} \in \hat{A}_{n}$, and $\mathbf{b}, \mathbf{c} \in \mathbb{G}(A)$, we have $\mathbf{a}+(\mathbf{b} \vee \mathbf{c})=(\mathbf{a}+\mathbf{b}) \vee(\mathbf{a}+\mathbf{c})$. The case $n=1$ is Lemma 7.22. Suppose that the statement holds for $n \in \mathbb{N}_{>0}$, and let us prove it for $n+1$. Let $\mathbf{a} \in \hat{A}_{n+1}$, and let $\mathbf{b}, \mathbf{c} \in \mathbb{G}(A)$. Then, there exists $\mathbf{a}^{\prime} \in \hat{A}_{n}$ and $\mathbf{x} \in \hat{A}$ such that $\mathbf{a}=\mathbf{a}^{\prime}+\mathbf{x}$ 
or $\mathbf{a}=\mathbf{x}+\mathbf{a}^{\prime}$. Since addition is commutative by Proposition 7.11 , these two conditions are equivalent. So,

$$
\begin{aligned}
\mathbf{a}+(\mathbf{b} \vee \mathbf{c}) & =\mathbf{x}+\mathbf{a}^{\prime}+(\mathbf{b} \vee \mathbf{c}) \\
& =\mathbf{x}+\left(\left(\mathbf{a}^{\prime}+\mathbf{b}\right) \vee\left(\mathbf{a}^{\prime}+\mathbf{c}\right)\right) \\
& =\left(\mathbf{x}+\mathbf{a}^{\prime}+\mathbf{b}\right) \vee\left(\mathbf{x}+\mathbf{a}^{\prime}+\mathbf{c}\right) \\
& =(\mathbf{a}+\mathbf{b}) \vee(\mathbf{a}+\mathbf{c}) .
\end{aligned}
$$

For a good sequence $\mathbf{a}=\left(a_{0}, a_{1}, a_{2}, \ldots\right)$ in an MV-monoidal algebra, set $\mathbf{a} \ominus 1:=\left(a_{1}, a_{2}, a_{3}, \ldots\right)$. The sequence $\mathbf{a} \ominus 1$ is a good sequence.

Proposition 7.24. For every $M V$-monoidal algebra $A$, the algebra $\mathbb{G}(A)$ is a positive-unital commutative $\ell$-monoid.

Proof. By Proposition 7.3, $\mathbb{G}(A)$ is a distributive lattice. By Propositions 7.11 and 7.20 and Remark 7.12, $\mathbb{G}(A)$ is a commutative monoid. By Proposition 7.23 , + distributes over $\wedge$ and $\vee$. Thus, $\mathbb{G}(A)$ is a commutative $\ell$-monoid (Axiom P0). Since the order in $\mathbb{G}(A)$ is pointwise (Remark 7.4), and 0 is the least element of $A$, we have Axiom P1, i.e., $\mathbf{0}$ is the least element of $\mathbb{G}(A)$. It is easy to see that $\left(a_{0}, a_{1}, a_{2}, \ldots\right)+\mathbf{1}=\left(1, a_{0}, a_{1}, a_{2}, \ldots\right)$. Therefore, we have Axiom P2, i.e., for all $\mathbf{a} \in \mathbb{G}(A)$, $\mathbf{a}+\mathbf{1} \ominus 1=\mathbf{a}$. For all $\mathbf{a} \in \mathbb{G}(A)$, we have $(\mathbf{a} \ominus 1)+\mathbf{1}=a \vee 1$, which establishes Axiom P3. By induction, one proves $n \mathbf{1}=(\underbrace{1, \ldots, 1}_{n \text { times }}, 0,0,0 \ldots)$. Since 1 is the maximum of $A$, we have Axiom $\mathrm{P} 4$, i.e., for all $\mathbf{a} \in \mathbb{G}(A)$, there exists $n \in \mathbb{N}$ such that $\mathbf{a} \leqslant n \mathbf{1}$.

Given a morphism of MV-monoidal algebras $f: A \rightarrow B$, we set

$$
\begin{aligned}
\mathbb{G}(f): \mathbb{G}(A) & \longrightarrow \mathbb{G}(B) \\
\left(x_{0}, x_{1}, x_{2}, \ldots\right) & \longmapsto\left(f\left(x_{0}\right), f\left(x_{1}\right), f\left(x_{2}\right), \ldots\right) .
\end{aligned}
$$

Lemma 7.25. For every morphism $f$ of $M V$-monoidal algebras, the function $\mathbb{G}(f)$ is a morphism of positive-unital commutative $\ell$-monoids.

Proof. Let us prove that $\mathbb{G}(f)$ preserves + . Set $\mathbf{z}:=\mathbf{x}+\mathbf{y}, \mathbf{u}:=f(\mathbf{z})$, and $\mathbf{w}:=f(\mathbf{x})+$ $f(\mathbf{y})$. Let $\mathbf{z}=\left(z_{0}, z_{1}, z_{2}, \ldots\right), \mathbf{u}=\left(u_{0}, u_{1}, u_{2}, \ldots\right)$ and $\mathbf{w}=\left(w_{0}, w_{1}, w_{2}, \ldots\right)$. We shall show $\mathbf{u}=\mathbf{w}$. For each $n \in \mathbb{N}$, we have

$$
z_{n}=\left(x_{0} \oplus y_{n}\right) \odot \cdots \odot\left(x_{n} \oplus y_{0}\right) \text {. }
$$

Thus,

$$
\begin{aligned}
u_{n} & =f\left(\left(x_{0} \oplus y_{n}\right) \odot \cdots \odot\left(x_{n} \oplus y_{0}\right)\right) \\
& =\left(f\left(x_{0}\right) \oplus f\left(y_{n}\right)\right) \odot \cdots \odot\left(f\left(x_{n}\right) \oplus f\left(y_{0}\right)\right) \\
& =f\left(\left(x_{0} \oplus y_{n}\right) \odot \cdots \odot\left(x_{n} \oplus y_{0}\right)\right) \\
& =w_{n} .
\end{aligned}
$$

Therefore, $\mathbb{G}(f)$ preserves + . Straightforward computations show that $\mathbb{G}(f)$ preserves also $0,1, \vee, \wedge$ and $\ominus$.

It is easy to see that $\mathbb{G}: M V M \rightarrow \mathrm{u} \ell \mathrm{M}^{+}$is a functor. 


\section{MV-monoidal algebras and positive cones are equivalent}

\subsection{The unit interval functor from positive cones}

Let $M$ be a positive-unital commutative $\ell$-monoid. We set $\mathbb{U}(M):=\{x \in M \mid$ $x \leqslant 1\}$; $\mathbb{U}$ stands for 'unit interval'. We endow $M$ with the operations of MV-monoidal algebra. The operations $\vee, \wedge, 0,1$ are defined by restriction. For $x, y \in \mathbb{U}(M)$, we set $x \oplus y:=(x+y) \wedge 1$ and $x \odot y:=(x+y) \ominus 1$. By the equivalence between $\mathrm{u} \ell \mathrm{M}^{+}$and $\mathrm{u} \ell \mathrm{M}$ (Theorem 4.16), and since $\tilde{\Gamma}\left(M^{\prime}\right)$ is an MV-monoidal algebra for every unital commutative $\ell$-monoid $M^{\prime}$ (Proposition 3.6), $\mathbb{U}(M)$ is an MV-monoidal algebra. Given a morphism $f: M \rightarrow N$ of positive-unital commutative $\ell$-monoids, we set $\mathbb{U}(f): \mathbb{U}(M) \rightarrow \mathbb{U}(N)$ as the restriction of $f$. This assignment establishes a functor $\mathbb{U}: \mathrm{u} \ell \mathrm{M}^{+} \rightarrow \mathrm{MVM}$.

\subsection{The unit}

For each MV-monoidal algebra $A$, consider the function

$$
\begin{aligned}
\eta_{A}^{1}: A & \longrightarrow \mathbb{U G}(A) \\
x & \longmapsto(x) .
\end{aligned}
$$

Proposition 8.1. For every $M V$-monoidal algebra $A$, the function $\eta_{A}^{1}: A \rightarrow$ $\mathbb{G}(A)$ is an isomorphism of $M V$-monoidal algebras.

Proof. The facts that $\eta_{A}^{1}$ is a bijection and that it preserves $0,1, \vee, \wedge$ are immediate. Let $x, y \in A$. Then, $(x)+(y)=(x \oplus y, x \odot y)$. Therefore

$$
\begin{aligned}
\eta_{A}^{1}(x) \oplus \eta_{A}^{1}(y) & =(x) \oplus(y)=((x)+(y)) \wedge \mathbf{1} \\
& =(x \oplus y, x \odot y) \wedge \mathbf{1}=(x \oplus y)=\eta_{A}^{1}(x \oplus y), \text { and } \\
\eta_{A}^{1}(x) \odot \eta_{A}^{1}(y) & =(x) \odot(y)=((x)+(y)) \ominus 1 \\
& =(x \oplus y, x \odot y) \ominus 1=(x \odot y)=\eta_{A}^{1}(x \odot y) .
\end{aligned}
$$

Proposition 8.2. $\eta^{1}: 1_{\mathrm{MVM}} \dot{\mathrm{UG}}$ is a natural transformation, i.e., for every morphism of MV-monoidal algebras $f: A \rightarrow B$, the following diagram commutes.

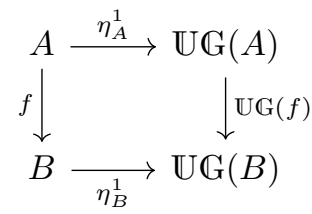

Proof. For every $x \in A$ we have

$$
\mathbb{U} \mathbb{G}(f)\left(\eta_{A}^{1}(x)\right)=\mathbb{U} \mathbb{G}(f)((x))=\mathbb{G}(f)((x))=(f(x))=\eta_{B}^{1}(f(x)) .
$$




\subsection{The counit}

For each positive-unital commutative $\ell$-monoid, we consider the function

$$
\begin{aligned}
\varepsilon_{M}^{1}: \mathbb{G U}(M) & \longrightarrow M \\
\left(x_{0}, \ldots, x_{n}\right) & \longmapsto x_{0}+\cdots+x_{n} .
\end{aligned}
$$

Our next goal, met in Proposition 8.13, is to prove that $\varepsilon_{M}^{1}$ is bijective; this will show that a positive-unital commutative $\ell$-monoid $M$ is in bijection with the set of good sequences in its unit interval $\mathbb{U}(M)$.

Lemma 8.3. Let $M$ be a positive-unital commutative $\ell$-monoid. For every $x \in$ $M$ and every $n \in \mathbb{N}$, we have

$$
x=(x \wedge n)+(x \ominus n) .
$$

Proof. We have

$$
(x \wedge n)+(x \ominus n)+n \stackrel{\text { Lemma }}{=}{ }^{4.14}(x \wedge n)+(x \vee n) \stackrel{\text { Lemma }}{=}{ }^{3.3} x+n .
$$

Since $n$ is cancellative by Lemma 4.2, it follows that $(x \wedge n)+(x \ominus n)=x$.

Lemma 8.4. Let $M$ be a positive-unital commutative $\ell$-monoid, let $x \in M$ and let $n \in \mathbb{N}$. If $x \leqslant n$, then $x \ominus n=0$.

Proof. By Lemma 4.14, we have

$$
(x \ominus n)+n=x \vee n=n=0+n .
$$

By Lemma 4.2, the element $n$ is cancellative: it follows that $x \ominus n=0$.

Lemma 8.5. Let $M$ be a positive-unital commutative $\ell$-monoid, let $x \in M$, and let $n, k \in \mathbb{N}$. Then,

$$
(x \ominus n) \wedge k=(x \wedge(n+k)) \ominus n .
$$

Proof. We have

$$
\begin{aligned}
((x \ominus n) \wedge k)+n & =((x \ominus n)+n) \wedge(k+n) & & (+ \text { distr. over } \wedge) \\
& =(x \vee n) \wedge(k+n) & & \text { (Lemma 4.14) } \\
& =(x \wedge(k+n)) \vee n & & \\
& =((x \wedge(k+n)) \ominus n)+n . & & \text { (Lemma 4.14) }
\end{aligned}
$$

Since $n$ is cancellative by Lemma 4.2, we have $(x \ominus n) \wedge k=(x \wedge(n+k)) \ominus n$.

Lemma 8.6. For every $x$ in a positive-unital commutative $\ell$-monoid, we have

$$
(x \wedge 1)+((x \ominus 1) \wedge 1)=x \wedge 2 .
$$

Proof. We have

$$
\begin{aligned}
(x & \wedge 1)+((x \ominus 1) \wedge 1)+1 & & \\
& =(x \wedge 1)+((x \ominus 1)+1) \wedge 2) & & (+ \text { distr. over } \wedge) \\
& =(x \wedge 1)+((x \vee 1) \wedge 2) & & (\text { Axiom P3 }) \\
& =((x \wedge 1)+(x \vee 1)) \wedge((x \wedge 1)+2) & & (+ \text { distr. over } \wedge)
\end{aligned}
$$




$$
\begin{aligned}
& =(x+1) \wedge(x+2) \wedge 3 \\
& (\text { Lemma 3.3, + distr. over } \wedge \text { ) } \\
& =(x+1) \wedge 3 \\
& =(x \wedge 2)+1 \text {. } \\
& (+ \text { distr. over } \wedge)
\end{aligned}
$$

Since 1 is cancellative by Lemma 4.2, we have $(x \wedge 1)+((x \ominus 1) \wedge 1)=x \wedge 2$.

Lemma 8.7. Let $M$ be a positive-unital commutative $\ell$-monoid, and let $x \in M$. Then, $(x \ominus 0, x \ominus 1, x \ominus 2, \ldots)$ is a good sequence in $\mathbb{U}(M)$.

Proof. For $n \in \mathbb{N}$, set $x_{n}:=x \ominus n$. Since $x \leqslant n$ for some $n \in \mathbb{N}$, the sequence $\left(x_{0}, x_{1}, x_{2}, \ldots\right)$ is eventually 0 by Lemma 8.4 . We have

$$
x_{n}+x_{n+1}=((x \ominus n) \wedge 1)+(((x \ominus n) \ominus 1) \wedge 1) \stackrel{\text { Lemma } 8.6}{=}(x \ominus n) \wedge 2 .
$$

Therefore,

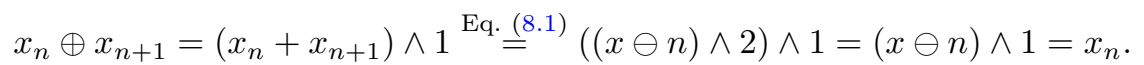

Moreover,

$$
\begin{aligned}
\left(x_{n} \odot x_{n+1}\right)+1 & =\left(\left(x_{n}+x_{n+1}\right) \ominus 1\right)+1 \\
& =\left(x_{n}+x_{n+1}\right) \vee 1 \\
& =((x \ominus n) \wedge 2) \vee 1 \\
& =((x \ominus n) \vee 1) \wedge 2 \\
& =(((x \ominus n) \ominus 1)+1) \wedge 2 \\
& =((x \ominus(n+1)) \wedge 1)+1 \\
& =x_{n+1}+1 .
\end{aligned}
$$

The element 1 is cancellative by Lemma 4.2 ; thus $x_{n} \odot x_{n+1}=x_{n+1}$.

Lemma 8.8. For every $m \in \mathbb{N}$ and every element $x$ of a positive-unital commutative $\ell$-monoid such that $x \leqslant m$, we have

$$
x=\sum_{n \in\{0, \ldots, m-1\}}(x \ominus n) \wedge 1 .
$$

Proof. We prove the statement by induction on $m \in \mathbb{N}$. If $m=0$, then $x=0$, and the assertion holds. Let us suppose that it holds for a fixed $m$, and let us prove that it holds for $m+1$. We recall that, by Lemma 8.5, we have $(x \ominus n) \wedge 1=(x \wedge(n+1)) \ominus n$. We have

$$
\begin{array}{rlrl}
x= & (x \wedge m)+(x \ominus m) & & \text { (Lemma 8.3) } \\
= & (x \wedge m)+((x \wedge(m+1)) \ominus n) & & (x \leqslant m+1) \\
= & \left(\sum_{n \in\{0, \ldots, m-1\}}((x \wedge m) \wedge(n+1)) \ominus n\right) & \\
& +((x \wedge(m+1)) \ominus n) & \\
= & \left(\sum_{n \in\{0, \ldots, m-1\}}(x \wedge(n+1)) \ominus n\right) &
\end{array}
$$




$$
\begin{aligned}
& +((x \wedge(m+1)) \ominus n) \\
= & \sum_{n \in\{0, \ldots, m\}}(x \wedge(n+1)) \ominus n .
\end{aligned}
$$

Remark 8.9. Let $M$ be a positive-unital commutative $\ell$-monoid, and let $x_{0}, x_{1} \in$ $\mathbb{U}(M)$. The pair $\left(x_{0}, x_{1}\right)$ is a good pair in $\mathbb{U}(M)$ if, and only if, $\left(x_{0}+x_{1}\right) \wedge 1=x_{0}$ and $\left(x_{0}+x_{1}\right) \ominus 1=x_{1}$. This is just unrolling the definitions.

Lemma 8.10. Let $M$ be a positive-unital commutative $\ell$-monoid. For every $m \in$ $\mathbb{N}$ and every good sequence $\left(x_{0}, \ldots, x_{m}\right)$ in $\mathbb{U}(M)$, we have

$$
\left(x_{0}+\cdots+x_{m}\right) \wedge 1=x_{0} .
$$

Proof. We prove the statement by induction on $m \in \mathbb{N}$. The case $m=0$ is trivial. The case $m=1$ holds by Remark 8.9. Suppose the statement holds for $m \in \mathbb{N}_{>0}$, and let us prove it holds for $m+1$. We have the following chain of equalities, the first of which is justified by the fact that $x_{0}+\cdots+x_{m-1}+1 \geqslant 1$.

$$
\begin{array}{rlrl}
\left(x_{0}\right. & \left.+\cdots+x_{m+1}\right) \wedge 1 & \\
& =\left(x_{0}+\cdots+x_{m+1}\right) \wedge\left(x_{0}+\cdots+x_{m-1}+1\right) \wedge 1 & \\
& =\left(x_{0}+\cdots+x_{m-1}+\left(\left(x_{m}+x_{m+1}\right) \wedge 1\right) \wedge 1\right. & & (+ \text { distr. over } \wedge) \\
& =\left(x_{0}+\cdots+x_{m-1}+x_{m}\right) \wedge 1 & & \text { (Remark 8.9) } \\
& =x_{0} . & & \text { (ind. hyp. })
\end{array}
$$

Lemma 8.11. Let $M$ be a positive-unital commutative $\ell$-monoid. For every $k \in$ $\mathbb{N}$ and every good sequence $\left(x_{0}, \ldots, x_{k}\right)$ in $\mathbb{U}(M)$, we have

$$
\left(x_{0}+\cdots+x_{k}\right) \ominus 1=x_{1}+\cdots+x_{k} .
$$

Proof. We prove this statement by induction on $k \in \mathbb{N}$. Equation (8.2) is equivalent to

$$
\left(\left(x_{0}+\cdots+x_{k}\right) \ominus 1\right)+1=1+x_{1}+\cdots+x_{k},
$$

i.e.,

$$
\left(x_{0}+\cdots+x_{k}\right) \vee 1=1+x_{1}+\cdots+x_{k} .
$$

The case $k=0$ is trivial. Let us suppose that the statement holds for a fixed $k \in \mathbb{N}$, and let us prove that it holds for $k+1$. We have

$$
\begin{aligned}
1+x_{1}+\cdots+x_{k+1} & =\left(1+x_{1}+\cdots+x_{k}\right)+x_{k+1} \\
& =\left(\left(x_{0}+\cdots+x_{k}\right) \vee 1\right)+x_{k+1} \\
& =\left(x_{0}+\cdots+x_{k}+x_{k+1}\right) \vee\left(1+x_{k+1}\right) \\
& =\left(x_{0}+\cdots+x_{k+1}\right) \vee\left(\left(x_{k}+x_{k+1}\right) \vee 1\right) \\
& =\left(\left(x_{0}+\cdots+x_{k+1}\right) \vee\left(x_{k}+x_{k+1}\right)\right) \vee 1 \\
& =\left(x_{0}+\cdots+x_{k+1}\right) \vee 1 .
\end{aligned}
$$


Lemma 8.12. Let $M$ be a positive-unital commutative $\ell$-monoid, let $m \in \mathbb{N}$, and let $\left(x_{0}, \ldots, x_{m}\right)$ and $\left(y_{0}, \ldots, y_{m}\right)$ be good sequences in $\mathbb{U}(M)$. If

$$
x_{0}+\cdots+x_{m}=y_{0}+\cdots+y_{m}
$$

then, for all $i \in\{0, \ldots, m\}, x_{i}=y_{i}$.

Proof. We prove the statement by induction on $m$. The case $m=0$ is trivial. Suppose that the statement holds for a fixed $m \in \mathbb{N}$, and let us prove it for $m+1$. By Lemma 8.10, we have

$$
x_{0}=\left(x_{0}+\cdots+x_{m+1}\right) \wedge 1=\left(y_{0}+\cdots+y_{m+1}\right) \wedge 1=y_{0} .
$$

By Lemma 8.11, we have

$$
\begin{aligned}
x_{1}+\cdots+x_{m+1} & =\left(x_{0}+x_{1}+\cdots+x_{m+1}\right) \ominus 1 \\
& =\left(y_{0}+y_{1}+\cdots+y_{m+1}\right) \ominus 1 \\
& =y_{1}+\cdots+y_{m+1} .
\end{aligned}
$$

By inductive hypothesis, for all $i \in\{1, \ldots, m+1\}, x_{i}=y_{i}$.

Proposition 8.13. Let $M$ be a positive-unital commutative $\ell$-monoid, and let $x \in M$. Then, there exists exactly one good sequence $\left(x_{0}, \ldots, x_{m}\right)$ in $\mathbb{U}(M)$ such that $x=x_{0}+\cdots+x_{m}$, given by

$$
x_{n}=(x \ominus n) \wedge 1 .
$$

In particular, the function $\varepsilon_{M}^{1}: \mathbb{G U}(M) \rightarrow M$ is bijective.

Proof. Lemmas 8.7 and 8.8 show that $x_{n}=(x \ominus n) \wedge 1$ works. Uniqueness is ensured by Lemma 8.12 .

Our next goal is to prove that $\varepsilon_{M}^{1}$ is a morphism of positive-unital commutative $\ell$-monoids (Proposition 8.18 below). We need some lemmas.

Lemma 8.14. Let $M$ be a positive-unital commutative $\ell$-monoid. For all good sequences $\left(x_{0}, \ldots, x_{m}\right)$ and $\left(y_{0}, \ldots, y_{m}\right)$ in $\mathbb{U}(M)$, we have

$$
\left(\left(\left(x_{0}+\cdots+x_{m}\right) \vee\left(y_{0}+\cdots+y_{m}\right)\right) \ominus n\right) \wedge 1=x_{n} \vee y_{n}
$$

and

$$
\left(\left(\left(x_{0}+\cdots+x_{m}\right) \wedge\left(y_{0}+\cdots+y_{m}\right)\right) \ominus n\right) \wedge 1=x_{n} \wedge y_{n}
$$

Proof. Let us prove Eq. (8.3). Set $x:=x_{0}+\cdots+x_{n}$, and $y:=y_{0}+\cdots+y_{n}$. By Proposition 8.13, we have $x_{n}=(x \ominus n) \wedge 1$ and $y_{n}=(y \ominus n) \wedge 1$. Adding $n$ on both sides of Eq. (8.3), we obtain the equivalent statement

$$
(x \vee y \vee n) \wedge(n+1)=((x \vee n) \wedge(n+1)) \vee((y \vee n) \wedge(n+1)),
$$

which holds by the distributivity laws. The proof of Eq. (8.4) is analogous.

Lemma 8.15. Let $M$ be a positive-unital commutative $\ell$-monoid, and let $x, y \in$ $M$ with $y \leqslant 1$. Set $x_{0}:=x \wedge 1$ and $x_{1}:=(x \ominus 1) \wedge 1$. Then,

$$
((x+y) \ominus 1) \wedge 1=x_{0} \odot\left(x_{1} \oplus y\right) .
$$


Proof. We have

$$
\begin{aligned}
x_{0}+x_{1} & =(x \wedge 1)+((x \ominus 1) \wedge 1) & & \\
& =((x \wedge 1)+(x \ominus 1)) \wedge((x \wedge 1)+1) & & (+ \text { distr. over } \wedge) \\
& =x \wedge(x+1) \wedge 2 & & \text { (Lemma 8.3) } \\
& =x \wedge 2 . & &
\end{aligned}
$$

Therefore, we have

$$
\begin{aligned}
x_{0} \odot\left(x_{1} \oplus y\right) & =\left(x_{0}+\left(\left(x_{1}+y\right) \wedge 1\right)\right) \ominus 1 & & (\text { def. of } \oplus \text { and } \odot) \\
& =\left(\left(x_{0}+x_{1}+y\right) \wedge\left(x_{0}+1\right)\right) \ominus 1 & & (+ \text { distr. over } \wedge) \\
& =((x+y) \wedge((x \wedge 1)+1)) \ominus 1 & & \\
& =((x+y) \wedge(x+1) \wedge 2) \ominus 1 & & (+ \text { distr. over } \wedge) \\
& =((x+y) \wedge 2) \ominus 1 & & \\
& =((x+y) \ominus 1) \wedge 1 . & & \text { (Lemma 8.5) }
\end{aligned}
$$

Lemma 8.16. Let $x$ and $y$ be elements of a positive-unital commutative $\ell$ monoid, let $n \in \mathbb{N} \backslash\{0\}$, and suppose $y \leqslant 1$. Then,

$$
(x+y) \ominus n=(x \ominus(n-1)+y) \ominus 1 .
$$

Proof. We have

$$
\begin{aligned}
(x & \ominus(n-1)+y) \ominus 1+n \\
& =(x \ominus(n-1)+y) \ominus 1+1+(n-1) \\
& =(x \ominus(n-1)+y) \vee 1+(n-1) \\
& =(x \ominus(n-1)+y+(n-1)) \vee n \\
& =((x \vee(n-1))+y) \vee n \\
& =(x+y) \vee(y+(n-1)) \vee n \\
& =(x+y) \vee n \\
& =((x+y) \ominus n)+n .
\end{aligned}
$$$$
(y \leqslant 1)
$$

Lemma 8.17. Let $M$ be a positive-unital commutative $\ell$-monoid, and let $x, y \in$ $M$ with $y \leqslant 1$. For every $n \in \mathbb{N}_{>0}$, we have

$$
((x+y) \ominus n) \wedge 1=((x \ominus(n-1)) \wedge 1) \odot(((x \ominus n) \wedge 1) \oplus y) .
$$

Proof. We have

$$
\begin{aligned}
& ((x+y) \ominus n) \wedge 1 \\
& \quad=(x \ominus(n-1)+y) \ominus 1 \\
& \quad=((x \ominus(n-1)) \wedge 1) \odot((((x \ominus(n-1)) \ominus 1) \wedge 1) \oplus y) \\
& \quad=((x \ominus(n-1)) \wedge 1) \odot(((x \ominus n) \wedge 1) \oplus y) .
\end{aligned}
$$

Proposition 8.18. For every positive-unital commutative $\ell$-monoid $M$, the function $\varepsilon_{M}^{1} \mathrm{GU}(M) \rightarrow M$ is a morphism of positive-unital commutative $\ell$-monoids. 
Proof. Clearly, $\varepsilon_{M}^{1}$ preserves 1 . Let us prove that $\varepsilon_{M}^{1}$ preserves $\vee$. Let $\mathbf{x}=$ $\left(x_{0}, \ldots, x_{m}\right)$ and $\mathbf{y}=\left(y_{0}, \ldots, y_{m}\right)$ be good sequences in $\mathbb{U}(M)$. We shall prove

$$
\left(x_{0}+\cdots+x_{m}\right) \vee\left(y_{0}+\cdots+y_{m}\right)=\left(x_{0} \vee y_{0}\right)+\cdots+\left(x_{m} \vee y_{m}\right) .
$$

By Proposition 7.2, $\left(x_{0} \vee y_{0}, \ldots, x_{m} \vee y_{m}\right)$ is a good sequence. By Proposition 8.13 , it is enough to show that, for every $n \in \mathbb{N}$,

$$
\left(\left(\left(x_{0}+\cdots+x_{m}\right) \vee\left(y_{0}+\cdots+y_{m}\right)\right) \ominus n\right) \wedge 1=x_{n} \vee y_{n}
$$

This holds by Lemma 8.14. Analogously, $\varepsilon_{M}^{1}$ preserves $\wedge$.

Let us prove that $\varepsilon_{M}^{1}$ preserves + . We prove, by induction on $n \in \mathbb{N}$, that, for all $m \in \mathbb{N}$, and for all $\left(x_{0}, \ldots, x_{m}\right)$ and $\left(y_{0}, \ldots, y_{n}\right)$ good sequences in $\mathbb{U}(M)$, we have

$$
\varepsilon_{M}^{1}\left(\left(x_{0}, \ldots, x_{m}\right)+\left(y_{0}, \ldots, y_{n}\right)\right)=x_{0}+\cdots+x_{m}+y_{0}+\cdots+y_{n} .
$$

Let us prove the base case $n=0$. Let $m \in \mathbb{N}$, let $\left(x_{0}, \ldots, x_{m}\right)$ be a good sequence in $\mathbb{U}(M)$, and let $y \in \mathbb{U}(M)$. Then, from the definition of sum of good sequences, we obtain that $\left(x_{0}, \ldots, x_{m}\right)+(y)$ is the good sequence $\left(z_{0}, \ldots, z_{m+1}\right)$ where, for every $k \in\{0, \ldots, m+1\}, z_{k}=x_{k-1} \odot\left(x_{k} \oplus y\right)$ (where, by convention, we set $x_{-1}=1$ ). By Lemma 8.17 and Proposition 8.13, for every $k \in \mathbb{N}$, we have

$$
\left(\left(x_{0}+\cdots+x_{m}+y\right) \ominus k\right) \wedge 1=x_{k-1} \odot\left(x_{k} \oplus y\right)=z_{k} .
$$

By Proposition 8.13, we have $z_{0}+\cdots+z_{m+1}=x_{0}+\cdots+x_{m}+y$; this settles the base case.

Let us suppose that the case $n$ holds, for a fixed $n \in \mathbb{N}$, and let us prove the case $n+1$. Let $m \in \mathbb{N}$, and let $\left(x_{0}, \ldots, x_{m}\right)$ and $\left(y_{0}, \ldots, y_{n+1}\right)$ be good sequences in $\mathbb{U}(M)$. Then

$$
\begin{aligned}
& \varepsilon_{M}^{1}\left(\left(x_{0}, \ldots, x_{m}\right)+\left(y_{0}, \ldots, y_{n+1}\right)\right) \\
& =\varepsilon_{M}^{1}\left(\left(x_{0}, \ldots, x_{m}\right)+\left(y_{0}, \ldots, y_{n}\right)+\left(y_{n+1}\right)\right) \\
& \left.\left.=\varepsilon_{M}^{1}\left(\left(x_{0}, \ldots, x_{m}\right)+\left(y_{0}, \ldots, y_{n}\right)\right)+\varepsilon_{M}^{1}\left(\left(y_{n+1}\right)\right) \quad \text { (base case }\right)\right) \\
& =\varepsilon_{M}^{1}\left(x_{0}, \ldots, x_{m}\right)+\varepsilon_{M}^{1}\left(y_{0}, \ldots, y_{n}\right)+y_{n+1} \quad \text { (ind. hyp.) } \\
& =x_{0}+\cdots+x_{m}+y_{0}+\cdots+y_{n}+y_{n+1} \text {. }
\end{aligned}
$$

Proposition 8.19. $\varepsilon^{1}: \mathbb{G U} \dot{\leftrightarrow} 1_{\mathrm{u} \mathrm{M}^{+}}$is a natural transformation, i.e., for every morphism of positive-unital commutative $\ell$-monoids $f: M \rightarrow N$, the following diagram commutes.

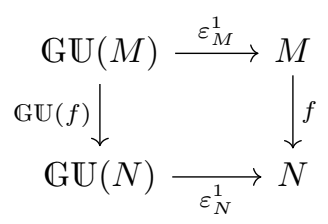


Proof. Let $\left(x_{0}, \ldots, x_{m}\right) \in \mathbb{G} \mathbb{U}(M)$. Then

$$
\begin{aligned}
\varepsilon_{N}^{1}\left(\mathbb{G U}(f)\left(x_{0}, \ldots, x_{m}\right)\right) & =\varepsilon_{N}^{1}\left(\mathbb{U}(f)\left(x_{0}\right), \ldots, \mathbb{U}(f)\left(x_{m}\right)\right) \\
& =\varepsilon_{N}^{1}\left(f\left(x_{0}\right), \ldots, f\left(x_{m}\right)\right) \\
& =f\left(x_{0}\right)+\cdots+f\left(x_{m}\right) \\
& =f\left(x_{0}+\cdots+x_{m}\right) \\
& =f\left(\varepsilon_{M}^{1}\left(x_{0}, \ldots, x_{m}\right)\right) .
\end{aligned}
$$

\subsection{The equivalence}

Theorem 8.20. The functors $\mathbb{U}: \mathrm{u} \ell \mathrm{M}^{+} \rightarrow \mathrm{MVM}$ and $\mathbb{G}: \mathrm{MVM} \rightarrow \mathrm{u} \ell \mathrm{M}^{+}$are quasi-inverses. Thus, the categories of unital commutative $\ell$-monoids and $M V$ monoidal algebras are equivalent.

Proof. By Propositions 8.1 and 8.2, the functors $1_{\mathrm{MVM}}: \mathrm{MVM} \rightarrow \mathrm{MVM}$ and $\mathbb{U G}: \mathrm{MVM} \rightarrow \mathrm{MVM}$ are naturally isomorphic. By Propositions 8.13, 8.18 and 8.19 , the functors $1_{\mathrm{u} \ell \mathrm{M}^{+}}: \mathrm{u} \ell \mathrm{M}^{+} \rightarrow \mathrm{u} \ell \mathrm{M}^{+}$and $\mathbb{G U}: \mathrm{u} \ell M^{+} \rightarrow \mathrm{u} \ell \mathrm{M}^{+}$are naturally isomorphic.

We are ready to prove the main result of the paper.

Theorem 8.21. The functor $\tilde{\Gamma}: \mathrm{u} \ell \mathrm{M} \rightarrow \mathrm{MVM}$ is an equivalence of categories.

Proof. The functor $\tilde{\Gamma}$ is the composite of $(-)^{+}$and $\mathbb{U}$, which are equivalences by Theorems 4.16 and 8.20 .

Notice that, by Theorems 4.16 and 8.20, a quasi-inverse of $\tilde{\Gamma}$ is given by the composite $\mathbb{T G}$.

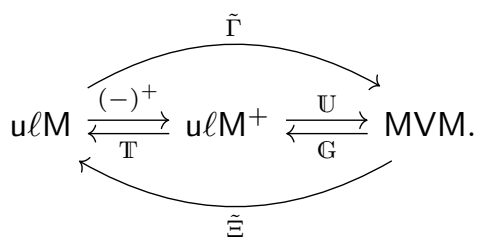

\section{Further research}

Some results about commutative $\ell$-monoids in the literature suggest similar ones for algebras in the language $\{\oplus, \odot, \vee, \wedge, 0,1\}$. For example, in [18] it is shown that the variety generated by $\langle\mathbb{R} ;+, \vee, \wedge\rangle$ does not admit a finite equational basis, and a countable basis is given in the same paper. Building on these results, the content of the present paper may possibly serve to obtain a nice equational basis for the variety generated by $\langle[0,1] ; \oplus, \odot, \vee, \wedge, 0,1\rangle$ which, we conjecture, is not finitely based; in particular, we conjecture that the variety of MVM-algebras is not generated by $[0,1]$.

We suspect that, from the results in the present paper, one may deduce a nice axiomatization of the quasi-variety generated by $\langle[0,1] ; \oplus, \odot, \vee, \wedge, 0,1\rangle$ and of the class of $\{\oplus, \odot, \vee, \wedge, 0,1\}$-subreducts of MV-algebras (conjecturally, these two classes coincide). 


\section{Acknowledgements}

The author is grateful to V. Marra for some comments and suggestions. Moreover, the author is indebted to the anonymous referee for several improvements, especially in the axiomatization of MV-monoidal algebras: for example, in a previous version of the paper, the boundedness of the lattice and the fact that 0 is absorbing for $\odot$ and 1 is absorbing for $\oplus$ were part of the axiomatization of MV-monoidal algebras, but the referee pointed out that they follow from the remaining axioms.

Funding Open access funding provided by Universitá degli Studi di Salerno within the CRUI-CARE Agreement.

Open Access. This article is licensed under a Creative Commons Attribution 4.0 International License, which permits use, sharing, adaptation, distribution and reproduction in any medium or format, as long as you give appropriate credit to the original author(s) and the source, provide a link to the Creative Commons licence, and indicate if changes were made. The images or other third party material in this article are included in the article's Creative Commons licence, unless indicated otherwise in a credit line to the material. If material is not included in the article's Creative Commons licence and your intended use is not permitted by statutory regulation or exceeds the permitted use, you will need to obtain permission directly from the copyright holder. To view a copy of this licence, visit http://creativecommons. org/licenses/by/4.0/.

Publisher's Note Springer Nature remains neutral with regard to jurisdictional claims in published maps and institutional affiliations.

\section{Appendix A. The equivalence restricts to lattice-ordered groups and MV-algebras}

In this section, we shortly hint at the fact that Mundici's equivalence follows from our main result.

We recall that a unital Abelian lattice-ordered group (unital Abelian $\ell$ group, for short) is an algebra $\langle G ;+, \vee, \wedge, 0,-, 1\rangle$ (arities $2,2,2,0,1,0)$ such that $\langle G ; \vee, \wedge\rangle$ is a distributive lattice, $\langle G ;+, 0,-\rangle$ is an Abelian group, + distributes over $\vee$ and $\wedge, 0 \leqslant 1$, and, for all $x \in M$, there exists $n \in \mathbb{N}$ such that $x \leqslant n 1$. We let $\mathrm{u} \ell \mathrm{G}$ denote the category of unital Abelian $\ell$-groups with homomorphisms. For all basic notions and results about lattice-ordered groups, we refer to [4]. In every unital Abelian $\ell$-group one defines the constant -1 as the additive inverse of 1 .

Remark A.1. It is not difficult to prove that the $\{+, \vee, \wedge, 0,1,-1\}$-reducts of unital Abelian $\ell$-groups are precisely the unital commutative $\ell$-monoids in which every element has an inverse. Moreover, the forgetful functor from $\mathrm{u} \ell \mathrm{G}$ to the category of $\{+, \vee, \wedge, 0,1,-1\}$-algebras is full and faithful.

We recall that an $M V$-algebra $\langle A ; \oplus, \neg, 0\rangle$ is a set $A$ equipped with a binary operation $\oplus$, a unary operation $\neg$ and a constant 0 such that $\langle A ; \oplus, 0\rangle$ 
is a commutative monoid, $\neg 0 \oplus x=\neg 0, \neg \neg x=x$ and $\neg(\neg x \oplus y) \oplus y=\neg(\neg y \oplus$ $x) \oplus x$. We let MV denote the category of MV-algebras with homomorphisms. For all basic notions and results about MV-algebras we refer to [7]. Via $\oplus, \neg$, 0 , one defines the operations $1:=\neg 0, x \odot y:=\neg(\neg x \oplus \neg y), x \vee y:=(x \odot \neg y) \oplus y$, and $x \wedge y:=x \odot(\neg x \oplus y)$.

Lemma A.2. Given an $M V$-algebra $\langle A ; \oplus, \neg, 0\rangle$, the algebra $\langle A ; \oplus, \odot, \vee, \wedge, 0,1\rangle$ is an $M V$-monoidal algebra.

Proof. Since $[0,1]$ generates the variety of MV-algebras [7, Theorem 2.3.5], it suffices to check that Axioms A1-A7 hold in $[0,1]$. This is the case because $\mathbb{R}$ is easily seen to be a unital commutative $\ell$-monoid and thus, by Proposition 3.6 , the unit interval $[0,1]$ is an MV-monoidal algebra.

Remark A.3. Using Lemma A.2, one proves that the $\{\oplus, \odot, \vee, \wedge, 0,1\}$-reducts of MV-algebras are the MV-monoidal algebras such that, for every $x$, there exists $y$ such that $x \oplus y=1$ and $x \odot y=0$. Moreover, the forgetful functor from MV to the category of $\{\oplus, \odot, \vee, \wedge, 0,1\}$-algebras is full and faithful.

Using Remarks A.1 and A.3, it is not too difficult to obtain the following.

Theorem A.4. The equivalence $\tilde{\Gamma}: \mathrm{u} \ell \mathrm{M} \rightarrow \mathrm{MVM}$ restricts to an equivalence between $\mathrm{u} \ell \mathrm{G}$ and $\mathrm{MV}$.

Remark A.5. In order to establish Theorem A.4 we used the axiom of choice. Precisely, we used the choice-based fact that $[0,1]$ generates the variety of MV-algebras to verify that every MV-algebra is an MV-monoidal algebra (Lemma A.2). If one proved without the axiom of choice that the axioms of MV-monoidal algebras are satisfied by every MV-algebra (and we suspect this to be possible), one would have a choice-free proof of Mundici's equivalence. The properties of lattices, Axiom A2, the distributivity of $\odot$ over $\vee$ and the distributivity of $\oplus$ over $\wedge$ were part of the original axiomatization of MV-algebras by Chang [5], which, as proved in [13] (see also [8, Section 2]), is equivalent to the modern one, presented here. A direct proof of Axioms A6 and A7 has been obtained with the help of Prover9, but we have not obtained proofs of the distributivity of the lattice, the distributivity of $\oplus$ over $\vee$, the distributivity of $\odot$ over $\wedge$, and Axioms A4 and A5.

\section{Appendix B. Subdirectly irreducible MV-monoidal algebras are totally ordered}

In this section, we prove that every subdirectly irreducible MV-monoidal algebra is totally ordered (Theorem B.3). We proceed in analogy with [19, Section 1]. Given an MV-monoidal algebra $A$, and a lattice congruence $\theta$ on $A$ such that $|A / \theta|=2$, we set

$$
\theta^{*}:=\{(a, b) \in A \times A \mid \forall x \in A(a \oplus x, b \oplus x) \in \theta \text { and }(a \odot x, b \odot x) \in \theta\} ;
$$


moreover, with $\mathbf{0}(\theta)$ and $\mathbf{1}(\theta)$ we denote the classes of the lattice congruence $\theta$ corresponding to smallest and greatest elements of the lattice $A / \theta$. An $M V M$ congruence on an MV-monoidal algebra $A$ is an equivalence relation on $A \times A$ that respects $\oplus, \odot, \vee, \wedge, 0,1$.

Lemma B.1. Let $A$ be an $M V$-monoidal algebra, and let $\theta$ be any lattice congruence on $A$ such that $|A / \theta|=2$. Then, $\theta^{*}$ is the greatest MVM-congruence contained in $\theta$.

Proof. It is not difficult to prove that $\theta^{*} \subseteq \theta$ and that $\theta^{*}$ contains every congruence contained in $\theta$.

We prove that $\theta^{*}$ is an MVM-congruence. The relation $\theta^{*}$ is an equivalence relation because $\theta$ is so.

In the following, let $a, a^{\prime}, b, b^{\prime} \in A$, and suppose $\left(a, a^{\prime}\right),\left(b, b^{\prime}\right) \in \theta^{*}$.

Let us prove $\left(a \vee b, a^{\prime} \vee b^{\prime}\right) \in \theta^{*}$. Let $x \in A$. Since $\left(a \oplus x, a^{\prime} \oplus x\right) \in \theta$, $\left(b \oplus x, b^{\prime} \oplus x\right) \in \theta$, and $\theta$ is a lattice congruence, we have $((a \oplus x) \vee(b \oplus$ $\left.x),\left(a^{\prime} \oplus x\right) \vee\left(b^{\prime} \oplus x\right)\right) \in \theta$, i.e., $\left((a \vee b) \oplus x,\left(a^{\prime} \vee b^{\prime}\right) \oplus x\right) \in \theta$. Analogously, $\left((a \vee b) \odot x,\left(a^{\prime} \vee b^{\prime}\right) \odot x\right) \in \theta$. This proves $\left(a \vee b, a^{\prime} \vee b^{\prime}\right) \in \theta^{*}$. Analogously, $\left(a \wedge b, a^{\prime} \wedge b^{\prime}\right) \in \theta^{*}$.

Let us prove $\left(a \oplus b, a^{\prime} \oplus b^{\prime}\right) \in \theta^{*}$. Let $x \in A$. We shall prove

$$
\left(a \oplus b \oplus x, a^{\prime} \oplus b^{\prime} \oplus x\right) \in \theta,
$$

and

$$
\left((a \oplus b) \odot x,\left(a^{\prime} \oplus b^{\prime}\right) \odot x\right) \in \theta .
$$

Since $\left(a, a^{\prime}\right) \in \theta^{*}$, we have $\left(a \oplus(b \oplus x), a^{\prime} \oplus(b \oplus x)\right) \in \theta$. Since $\left(b, b^{\prime}\right) \in \theta^{*}$, we have $\left(b \oplus\left(a^{\prime} \oplus x\right), b^{\prime} \oplus\left(a^{\prime} \oplus x\right)\right) \in \theta$. Hence, by transitivity of $\theta$, we have $\left(a \oplus b \oplus x, a^{\prime} \oplus b^{\prime} \oplus x\right) \in \theta$, and so Eq. (B.1) is proved.

Let us prove Eq. (B.2). By transitivity of $\theta$, it is enough to prove

$$
\left((a \oplus b) \odot x,\left(a^{\prime} \oplus b\right) \odot x\right) \in \theta,
$$

and

$$
\left(\left(a^{\prime} \oplus b\right) \odot x,\left(a^{\prime} \oplus b^{\prime}\right) \odot x\right) \in \theta .
$$

Let us prove Eq. (B.3). Suppose, by way of contradiction, $\left((a \oplus b) \odot x,\left(a^{\prime} \oplus\right.\right.$ $b) \odot x) \notin \theta$. Then, without loss of generality, we may assume $(a \oplus b) \odot x \in \mathbf{0}(\theta)$ and $\left(a^{\prime} \oplus b\right) \odot x \in \mathbf{1}(\theta)$. We have $\mathbf{1}(\theta) \ni\left(a^{\prime} \oplus b\right) \odot x \leqslant x$; thus $x \in \mathbf{1}(\theta)$. We have

$$
\underbrace{(a \oplus b) \odot x}_{\in \mathbf{0}(\theta)}=\sigma(a, b, x) \wedge \underbrace{x}_{\in \mathbf{1}(\theta)},
$$

and thus $\sigma(a, b, x) \in \mathbf{0}(\theta)$. We have

$$
\underbrace{\left(a^{\prime} \oplus b\right) \odot x}_{\in \mathbf{1}(\theta)}=\sigma\left(a^{\prime}, b, x\right) \wedge \underbrace{x}_{\in \mathbf{1}(\theta)},
$$

and thus $\sigma\left(a^{\prime}, b, x\right) \in \mathbf{1}(\theta)$. We have

$$
\mathbf{0}(\theta) \ni \sigma(a, b, x)=(a \odot(b \oplus x)) \oplus(b \odot x) \geqslant a \odot(b \oplus x),
$$


and thus $a \odot(b \oplus x) \in \mathbf{0}(\theta)$. Since $\left(a, a^{\prime}\right) \in \theta^{*}$, it follows that $a^{\prime} \odot(b \oplus x) \in \mathbf{0}(\theta)$. We have

$$
\underbrace{a^{\prime} \odot(b \oplus x)}_{\in \mathbf{0}(\theta)}=a^{\prime} \wedge \underbrace{\sigma\left(a^{\prime}, b, x\right)}_{\in \mathbf{1}(\theta)} .
$$

Therefore, $a^{\prime} \in \mathbf{0}(\theta)$. We have

$$
\mathbf{1}(\theta) \ni \sigma\left(a^{\prime}, b, x\right)=\left(a^{\prime} \oplus(b \odot x)\right) \odot(b \oplus x) \leqslant a^{\prime} \oplus(b \odot x),
$$

and thus $a^{\prime} \oplus(b \odot x) \in \mathbf{1}(\theta)$. Since $\left(a, a^{\prime}\right) \in \theta^{*}$, it follows that $a \oplus(b \odot x) \in \mathbf{1}(\theta)$. We have

$$
\underbrace{a \oplus(b \odot x)}_{\in \mathbf{1}(\theta)}=a \vee \underbrace{\sigma(a, b, x)}_{\in \mathbf{0}(\theta)} .
$$

Therefore, $a \in \mathbf{1}(\theta)$. Thus, $a \in \mathbf{1}(\theta)$ and $a^{\prime} \in \mathbf{0}(\theta)$, and this contradicts $\left(a, a^{\prime}\right) \in \theta^{*} \subseteq \theta$. In conclusion, Eq. (B.3) holds, and analogously for Eq. (B.4). By transitivity of $\theta$, Eq. (B.2) holds. Thus, $\left(a \oplus b, a^{\prime} \oplus b^{\prime}\right) \in \theta^{*}$. Analogously, $\left(a \odot b, a^{\prime} \odot b^{\prime}\right) \in \theta^{*}$.

We denote with $\Delta$ the identity relation $\{(s, s) \mid s \in A\}$.

Lemma B.2. If $A$ is a subdirectly irreducible $M V$-monoidal algebra, then there exists a lattice congruence $\theta$ on $A$ such that $|A / \theta|=2$ and $\theta^{*}=\Delta$.

Proof. Since $A$ is distributive as a lattice, it can be decomposed into a subdirect product of two-element lattices. Let $\left\{\theta_{i}\right\}_{i \in I}$ be the set of lattice congruences of $A$ corresponding with such a decomposition. Then $\bigcap_{i \in I} \theta_{i}=\Delta$. By Lemma B.1, each $\theta_{i}^{*}$ is an MVM-congruence, and $\Delta \subseteq \theta_{i}^{*} \subseteq \theta_{i}$. Therefore we have $\bigcap_{i \in I} \theta_{i}^{*}=\Delta$, and the fact that $A$ is subdirectly irreducible implies $\theta_{j}^{*}=\Delta$ for some $j \in I$.

Theorem B.3. Every subdirectly irreducible MV-monoidal algebra is totally ordered.

Proof. Let $A$ be a subdirectly irreducible MV-monoidal algebra. Lemma B.2 entails that there exists a lattice congruence $\theta$ on $A$ such that $|A / \theta|=2$ and such that $\theta^{*}=\Delta$, i.e., for all distinct $a, b \in A$, there exists $x \in A$ such that $(a \oplus x, b \oplus x) \notin \theta$ or $(a \odot x, b \odot x) \notin \theta$.

Let $a, b \in A$. We shall prove that either $a \leqslant b$ or $b \leqslant a$ holds. Suppose, by way of contradiction, that this is not the case, i.e., $a \wedge b \neq a$ and $a \wedge b \neq$ $b$. Since $a \wedge b \neq a$, there exists $x \in A$ such that $((a \wedge b) \oplus x, a \oplus x) \notin \theta$ or $((a \wedge b) \odot x, a \odot x) \notin \theta$. Since $a \wedge b \neq b$, there exists $y \in A$ such that $((a \wedge b) \oplus y, b \oplus y) \notin \theta$ or $((a \wedge b) \odot y, b \odot y) \notin \theta$. We have four cases.

(1) Case $((a \wedge b) \oplus x, a \oplus x) \notin \theta$ and $((a \wedge b) \oplus y, b \oplus y) \notin \theta$.

Since $a \wedge b \leqslant a$ and $a \wedge b \leqslant b$, we have $(a \wedge b) \oplus x \in \mathbf{0}(\theta), a \oplus x \in \mathbf{1}(\theta)$, $(a \wedge b) \oplus y \in \mathbf{0}(\theta)$, and $b \oplus y \in \mathbf{1}(\theta)$. Then,

$$
\begin{aligned}
\mathbf{0}(\theta) & \ni((a \wedge b) \oplus x) \vee((a \wedge b) \oplus y) & & \\
& =(a \wedge b) \oplus(x \vee y) & & (\oplus \text { distr. over } \vee) \\
& =(a \oplus(x \vee y)) \wedge(b \oplus(x \vee y)) & & (\oplus \text { distr. over } \wedge)
\end{aligned}
$$




$$
\begin{aligned}
& \geqslant(a \oplus x) \wedge(b \oplus y) \\
& \in \mathbf{1}(\theta),
\end{aligned}
$$

which is a contradiction.

(2) The case $((a \wedge b) \odot x, a \odot x) \notin \theta$ and $((a \wedge b) \odot y, b \odot y) \notin \theta$ is analogous to item 1.

(3) Case $((a \wedge b) \oplus x, a \oplus x) \notin \theta$ and $((a \wedge b) \odot y, b \odot y) \notin \theta$.

Since $a \wedge b \leqslant a$, we have $(a \wedge b) \oplus x \in \mathbf{0}(\theta)$, and $a \oplus x \in \mathbf{1}(\theta)$. Therefore,

$$
\mathbf{0}(\theta) \ni(a \wedge b) \oplus x=\underbrace{(a \oplus x)}_{\in \mathbf{1}(\theta)} \wedge(b \oplus x) .
$$

Hence $b \oplus x \in \mathbf{0}(\theta)$, which implies $b \in \mathbf{0}(\theta)$, which implies $(a \wedge b) \odot y \in \mathbf{0}(\theta)$ and $b \odot y \in \mathbf{0}(\theta)$, which contradicts $((a \wedge b) \odot y, b \odot y) \notin \theta$.

(4) The case $((a \wedge b) \odot x, a \odot x) \notin \theta$ and $((a \wedge b) \oplus y, b \oplus y) \notin \theta$ is analogous to item 3 .

In any case, we are led to a contradiction.

\section{Appendix C. Good pairs in subdirectly irreducible MV-monoidal algebras}

The goal of this section-met in Corollary C.6-is to show that good sequences in a subdirectly irreducible MV-monoidal algebra are of the form $(1, \ldots, 1, x, 0,0, \ldots)$.

Notation C.1. Let $A$ be an MV-monoidal algebra and let $x \in A$. For $a, a^{\prime} \in A$, set $a \sim_{\perp}^{x} a^{\prime}$ if, and only if, there exist $n, m \in \mathbb{N}$ such that

$$
a \oplus(\underbrace{x \oplus \cdots \oplus x}_{n \text { times }}) \geqslant a^{\prime}, \quad \quad a^{\prime} \oplus(\underbrace{x \oplus \cdots \oplus x}_{m \text { times }}) \geqslant a .
$$

Moreover, set $a \sim_{x}^{\top} a^{\prime}$ if, and only if, there exist $n, m \in \mathbb{N}$ such that

$$
b \odot(\underbrace{x \odot \cdots \odot x}_{n \text { times }}) \leqslant b^{\prime}, \quad \quad b^{\prime} \odot(\underbrace{x \odot \cdots \odot x}_{m \text { times }}) \leqslant b .
$$

It is not difficult to prove the following.

Lemma C.2. For every $M V$-monoidal algebra $A$ and every $x \in A$, the relation $\sim_{\perp}^{x}$ is the smallest MVM-congruence $\sim$ on $A$ such that $x \sim 0$, and the relation $\sim_{x}^{\top}$ is the smallest MVM-congruence $\sim$ on A such that $x \sim 1$.

Lemma C.3. Let $A$ be an MV-monoidal algebra, let $\left(x_{0}, x_{1}\right)$ be a good pair in $A$, and let $a, b \in A$ be such that $a \leqslant b \oplus x_{1}$ and $a \odot x_{0} \leqslant b$. Then, $a \leqslant b$.

Proof. Let us first deal with the case $b \leqslant a$; under this hypothesis, we shall prove $a=b$. Since $a \leqslant b \oplus x_{1}$, we have

$$
a \odot x_{0} \leqslant\left(b \oplus x_{1}\right) \odot x_{0} \stackrel{\text { Lemma }}{=} 6.9 \sigma\left(b, x_{0}, x_{1}\right) .
$$

Since $a \odot x_{0} \leqslant \sigma\left(b, x_{0}, x_{1}\right)$ and $a \odot x_{0} \leqslant b$, we have

$$
a \odot x_{0} \leqslant b \wedge \sigma\left(b, x_{0}, x_{1}\right)=b \odot\left(x_{0} \oplus x_{1}\right)=b \odot x_{0} .
$$


Since $b \leqslant a$, we have $b \odot x_{0} \leqslant a \odot x_{0}$. Hence, $a \odot x_{0}=b \odot x_{0}$. Analogously, $a \oplus x_{1}=b \oplus x_{1}$. Hence

$$
\sigma\left(a, x_{0}, x_{1}\right) \stackrel{\text { Lemma }}{=} 6.9\left(a \odot x_{0}\right) \oplus x_{1}=\left(b \odot x_{0}\right) \oplus x_{1} \stackrel{\text { Lemma }}{=} 6.9 \sigma\left(b, x_{0}, x_{1}\right) .
$$

Set $s:=\sigma\left(a, x_{0}, x_{1}\right)=\sigma\left(b, x_{0}, x_{1}\right)$. To prove $a=b$ it is enough to prove $a \vee s=$ $b \vee s$ and $a \wedge s=b \wedge s$. We have

$$
a \vee s=a \oplus\left(x_{0} \odot x_{1}\right)=a \oplus x_{1}=b \oplus x_{1}=b \oplus\left(x_{0} \odot x_{1}\right)=b \vee s,
$$

and

$$
a \wedge s=a \odot\left(x_{0} \oplus x_{1}\right)=a \odot x_{0}=b \odot x_{0}=b \odot\left(x_{0} \oplus x_{1}\right)=b \wedge s .
$$

Hence, $a=b$.

If we do not assume $b \leqslant a$, we may replace $b$ with $a \wedge b$, because

$$
a \leqslant\left(a \oplus x_{1}\right) \wedge\left(b \oplus x_{1}\right)=(a \wedge b) \oplus x_{1},
$$

and $a \odot x_{0} \leqslant a \wedge b$. Since $a \wedge b \leqslant a$, by the previous part we have $a \wedge b=a$, i.e., $a \leqslant b$.

Theorem C.4. Let $A$ be a subdirectly irreducible $M V$-monoidal algebra. Then, for all $x, y \in A$, either $x \oplus y=1$ or $x \odot y=0$.

Proof. Set $u:=x \oplus y$ and $v:=x \odot y$. We claim $\sim_{\perp}^{v} \cap \sim_{u}^{\top}=\Delta$. Indeed, let $a, b \in A$ with $a \sim_{\perp}^{v} b$ and $a \sim_{u}^{\top} b$. Then, there exist $n, m \in \mathbb{N}$ such that

$$
a \leqslant b \oplus(\underbrace{v \oplus \cdots \oplus v}_{m \text { times }}), \quad a \odot(\underbrace{u \odot \cdots \odot u}_{n \text { times }}) \leqslant b .
$$

Since $(u, v)$ is a good pair by Lemma 6.10 , then, by Lemma 7.5 , also

$$
(u \odot \cdots \odot u, v \oplus \cdots \oplus v)
$$

is so. By Lemma C.3, $a \leqslant b$. Analogously, $b \leqslant a$, and therefore $a=b$. This settles the claim $\sim_{\perp}^{v} \cap \sim_{u}^{\top}=\Delta$. By Lemma C.2, $\sim_{\perp}^{x \odot y}$ and $\sim_{x \oplus y}^{\top}$ are MVMcongruences, $x \odot y \sim_{\perp}^{x \odot y} 0$ and $x \oplus y \sim_{x \oplus y}^{\top} 1$. Since $A$ is subdirectly irreducible, either $\sim_{\perp}^{v}=\Delta$ or $\sim_{u}^{\top}=\Delta$. In the former case we have $v=0$, i.e. $x \odot y=0$; in the latter one we have $u=1$, i.e. $x \oplus y=1$.

Corollary C.5. Let $\left(x_{0}, x_{1}\right)$ be a good pair in a subdirectly irreducible $M V$ monoidal algebra. Then, either $x_{0}=1$ or $x_{1}=0$.

Corollary C.6. Every good sequence in a subdirectly irreducible MV-monoidal algebra is of the form $(1, \ldots, 1, x, 0,0, \ldots)$.

\section{Appendix D. Independence of the axioms}

With the help of Mace4 [15], we verified that, once one of the equivalent Axioms A4 and A5 is removed, the axioms of MV-monoidal algebras are independent. In particular, each of the following properties does not follow from the conjunction of the other ones.

(1) $\vee$ and $\wedge$ satisfy the axioms of a lattice. 
(2) $\vee$ left- and right-distributes over $\wedge$ and $\wedge$ left- and right-distributes over $\checkmark$.

(3) $\oplus$ is associative.

(4) $\odot$ is associative.

(5) $\oplus$ is commutative.

(6) $\odot$ is commutative.

(7) $x \oplus 0=x$ and $0 \oplus x=x$.

(8) $x \odot 1=x$ and $1 \odot x=x$.

(9) $\oplus$ left- and right-distributes over $\vee$.

(10) $\odot$ left- and right-distributes over $\wedge$.

(11) $\oplus$ left- and right-distributes over $\wedge$.

(12) $\odot$ left- and right-distributes over $\vee$.

(13) $(x \odot y) \oplus((x \oplus y) \odot z)=(x \oplus(y \odot z)) \odot(y \oplus z)$ and $(x \oplus y) \odot((x \odot y) \oplus z)=(x \odot(y \oplus z)) \oplus(y \odot z)$.

(14) $(x \odot y) \oplus z=((x \oplus y) \odot((x \odot y) \oplus z)) \vee z$ and $x \oplus(y \odot z)=x \vee((x \oplus(y \odot z)) \odot(y \oplus z))$.

(15) $(x \oplus y) \odot z=((x \odot y) \oplus((x \oplus y) \odot z)) \wedge z$ and $x \odot(y \oplus z)=x \wedge((x \odot(y \oplus z)) \oplus(y \odot z))$.

\section{References}

[1] Abbadini, M.: The dual of compact ordered spaces is a variety. Theory Appl. Categ. 34, 1401-1439 (2019)

[2] Abbadini, M.: On the axiomatisability of the dual of compact ordered spaces. Ph.D. Thesis, University of Milan (2021)

[3] Abbadini, M., Reggio, L.: On the axiomatisability of the dual of compact ordered spaces. Appl. Categ. Struct. 28, 921-934 (2020)

[4] Bigard, A., Keimel, K., Wolfenstein, S.: Groupes et Anneaux Réticulés. Lecture Notes in Mathematics, vol. 608. Springer, Berlin (1977)

[5] Chang, C.C.: Algebraic analysis of many valued logics. Trans. Am. Math. Soc. 88, 467-490 (1958)

[6] Choudhury, A.C.: The doubly distributive $m$-lattice. Bull. Calcutta Math. Soc. 49, 71-74 (1957)

[7] Cignoli, R.L.O., D'Ottaviano, I.M.L., Mundici, D.: Algebraic Foundations of Many-Valued Reasoning, Trends in Logic-Studia Logica Library, vol. 7. Kluwer Academic Publishers, Dordrecht (2000)

[8] Cignoli, R., Mundici, D.: An elementary proof of Chang's completeness theorem for the infinite-valued calculus of Łukasiewicz. Studia Logica 58, 79-97 (1997)

[9] Duskin, J.: Variations on Beck's tripleability criterion. In: Reports of the Midwest Category Seminar. III, pp. 74-129. Springer, Berlin (1969) 
[10] Hofmann, D., Neves, R., Nora, P.: Generating the algebraic theory of $C(X)$ : the case of partially ordered compact spaces. Theory Appl. Categ. 33, 276-295 (2018)

[11] Isbell, J.: Generating the algebraic theory of $C(X)$. Algebra Universalis 15, 153$155(1982)$

[12] Mac Lane, S.: Categories for the Working Mathematician. Graduate Texts in Mathematics, vol. 5, 2nd edn. Springer, New York (1998)

[13] Mangani, P.: Su certe algebre connesse con logiche a più valori. Boll. Un. Mat. Ital. 8, 68-78 (1973). Italian

[14] Marra, V., Reggio, L.: Stone duality above dimension zero: axiomatising the algebraic theory of $\mathrm{C}(X)$. Adv. Math. 307, 253-287 (2017)

[15] McCune, W.: Prover9 and Mace4 (2005-2010). http://www.cs.unm.edu/ $\sim$ mccune/prover9/

[16] Mundici, D.: Interpretation of AF $C^{*}$-algebras in Łukasiewicz sentential calculus. J. Funct. Anal. 65, 15-63 (1986)

[17] Nachbin, L.: Topology and Order. Translated from the Portuguese by Lulu Bechtolsheim. Van Nostrand Mathematical Studies, vol. 4. D. Van Nostrand Co., Inc., Princeton, Toronto (1965)

[18] Repnitskii, V.B.: Bases of identities of varieties of lattice-ordered semigroups. Algebra Logika 22, 649-665 (1983)

[19] Repnitzkii, V.B.: On subdirectly irreducible lattice-ordered semigroups. Semigroup Forum 29, 277-318 (1984)

[20] Stone, M.H.: Applications of the theory of Boolean rings to general topology. Trans. Am. Math. Soc. 41, 375-481 (1937)

[21] Yosida, K.: On vector lattice with a unit. Proc. Imp. Acad. Tokyo 17, 121-124 (1941)

Marco Abbadini

Department of Mathematics

University of Salerno

Via Giovanni Paolo II, 132

Fisciano SA

Italy

e-mail: mabbadini@unisa.it

URL: http://logica.dipmat.unisa.it/marcoabbadini/

Received: 31 July 2019.

Accepted: 12 May 2021. 